\title{
The Motivic Spectral Sequence
}

\author{
Daniel R. Grayson ${ }^{\star}$ \\ University of Illinois at Urbana-Champaign, dan@math.uiuc.edu
}

Summary. We give an overview of the search for a motivic spectral sequence: a spectral sequence connecting algebraic K-theory to motivic cohomology that is analogous to the Atiyah-Hirzebruch spectral sequence that connects topological Ktheory to singular cohomology.

\section{Introduction}

In this chapter we explain the Atiyah-Hirzebruch spectral sequence that relates topological K-theory to singular cohomology and try to motivate the search for a motivic version. In the time since [18] appeared, which concerns motivation for such a motivic spectral sequence, many authors have produced results in this direction. We describe the Bloch-Lichtenbaum spectral sequence [8] for the spectrum of a field together with the Friedlander-Suslin and Levine extensions $[12,28]$ to the global case for a smooth variety over a field. We explain the Goodwillie-Lichtenbaum idea involving tuples of commuting automorphisms and the theorem [19] that uses it to produce a motivic spectral sequence for an affine regular noetherian scheme, unfortunately involving certain non-standard motivic cohomology groups. We present Suslin's result [41], that, for smooth varieties over a field, these non-standard motivic cohomology groups are isomorphic to the standard groups. We sketch Voevodsky's approach via the slice filtration $[43,47,49]$, much of which remains conjectural. Finally, we sketch Levine's recent preprint [29], which gives a novel approach that yields a spectral sequence for smooth varieties over a field and makes it extremely clear which formal properties of $K$-theory are used in the proof. At this point we refer the reader also to [11] where a similar spectral sequence is developed for semi-topological $K$-theory.

The importance of the motivic spectral sequence lies in its applications. Important work of Voevodsky $[42,44,48]$ makes motivic cohomology amenable

\footnotetext{
* This work has been supported by NSF grant DMS 99-70085.
} 
to computation, and the motivic spectral sequence is the route by which those computations can be used to compute algebraic $K$-groups. For such applications, see, for example, [51, 40, 39, 34, 35, 22, 36, 24].

The main open question now seems to be how to handle a general noetherian regular scheme, such as those that arise in number theory. In all the papers cited, except for [18], the strongest results are true only for smooth varieties over a field.

Many fine papers have been written on this topic - any difference in the depths to which I manage to expose them are due more to personal limitations of time and ability than to any judgment of their relative importance.

\section{Contents}

$1 \quad$ Algebraic K-theory and cohomology $\ldots \ldots \ldots \ldots \ldots \ldots, 2$

2 Topological K-theory and cohomology ............... 4

3 The motivic spectral sequence $\ldots \ldots \ldots \ldots \ldots \ldots \ldots \ldots, 6$

4 Filtrations as a source of spectral sequences........... 7

5 Commuting automorphisms .................... 9

6 Cancellation and comparison with motivic cohomology .... 15

7 Higher Chow groups and a motivic spectral sequence ..... 19

8 Extension to the global case ..................... 22

9 The slice filtration $\ldots \ldots \ldots \ldots \ldots \ldots \ldots \ldots \ldots \ldots \ldots \ldots \ldots \ldots \ldots \ldots \ldots, 24$

10 Filtrations for general cohomology theories ............ 25

References .................................... 27

\section{Algebraic K-theory and cohomology}

The Riemann-Roch theorem for a complete nonsingular algebraic curve $X$ over a field $k$ relates the degree of a line bundle $\mathcal{L}$ to the dimension of its space $\Gamma(X, \mathcal{L})$ of global sections. The functor $\Gamma(X,-)$ is not an exact functor, so what really enters into the theorem (on one side of an equation) is the Euler characteristic, defined by $\chi(\mathcal{L}):=\sum_{i}(-1)^{i} \operatorname{dim} H^{i}(X, \mathcal{L})$. The Euler characteristic is additive in the sense that $\chi(\mathcal{E})=\chi\left(\mathcal{E}^{\prime}\right)+\chi\left(\mathcal{E}^{\prime \prime}\right)$ whenever $0 \rightarrow \mathcal{E}^{\prime} \rightarrow \mathcal{E} \rightarrow \mathcal{E}^{\prime \prime} \rightarrow 0$ is an exact sequence of (locally free) coherent sheaves on $X$. The natural way to prove formulas relating one additive function on coherent sheaves to another is to work with the universal target for such additive functions, so Grothendieck defined $K_{0}(X)$ to be the abelian group generated by the isomorphism classes $[\mathcal{E}]$ of locally free coherent sheaves on $X$, 
with relations $[\mathcal{E}]=\left[\mathcal{E}^{\prime}\right]+\left[\mathcal{E}^{\prime \prime}\right]$ for each exact sequence as before. Alternatively, one defines $K_{0}^{\prime}(X)$ by using all coherent sheaves, not just the locally free ones. Tensor product of coherent sheaves makes $K_{0}(X)$ into a ring and $K_{0}^{\prime}(X)$ into a module over it. For a nonsingular quasi-projective algebraic variety $X$, the natural map $K_{0}(X) \rightarrow K_{0}^{\prime}(X)$ is an isomorphism, because a coherent sheaf has a resolution of finite length by locally free coherent sheaves.

The group $K_{0}^{\prime}(X)$ has a filtration whose $i$-th member $F^{i} K_{0}^{\prime}(X)$ is the subgroup generated by the classes of coherent sheaves whose support has codimension at least $i$. The ring $K_{0}(X)$ has a more complicated filtration with members $F_{\gamma}^{i} K_{0}(X)$ called the $\gamma$-filtration (formerly, the $\lambda$-filtration) [14, III, $\S 1$ ], arising from a detailed consideration of the way exterior powers of vector bundles behave with respect to short exact sequences of bundles. Let $G r_{\gamma}^{i} K_{0}(X)$ and $G r^{i} K_{0}^{\prime}(X)$ denote the associated graded groups. When $X$ is a nonsingular quasi-projective algebraic variety the map $K_{0}(X) \rightarrow K_{0}^{\prime}(X)$ respects the filtration [21, X 1.3.2] [14, VI 5.5], and the induced map $G r_{\gamma}^{i} K_{0}(X)_{\mathbb{Q}} \rightarrow$ $G r^{i} K_{0}^{\prime}(X)_{\mathbb{Q}}$ is an isomorphism [21, VII 4.11, X 1.3.2], where $(-)_{\mathbb{Q}}$ denotes tensoring with the field of rational numbers.

The Grothendieck group $K_{0}(X)$ was an essential tool in Grothendieck's proof of the Grothendieck-Riemann-Roch theorem [21], which extended the Riemann-Roch theorem for curves to nonsingular varieties of any dimension. The other important ingredient was the Chow ring. An algebraic cycle on $X$ of codimension $i$ is a formal linear combination of closed subvarieties $Z$ (reduced and irreducible) of $X$ of codimension $i$. The group of such cycles is denoted by $\mathcal{Z}^{i}(X)$. Such cycles arise naturally when intersecting two subvarieties as a way of keeping track of the multiplicities with which the components of the intersection should be counted. Two algebraic cycles are called linearly equivalent if they are members of the same family parametrized algebraically by the points of the affine line $\mathbb{A}^{1}$. The automorphism group of a projective space contains plenty of straight lines, so linear equivalence of algebraic cycles allows pairs of cycles whose intersection doesn't have the maximal possible codimension to be moved to achieve that condition. Let $C H^{i}(X)$ denote the group of codimension $i$ algebraic cycles on $X$ modulo linear equivalence; it is the degree $i$ component of a graded ring $C H(X)$ whose multiplication comes from intersection of cycles.

One consequence of the Grothendieck-Riemann-Roch theorem is that when $X$ is a nonsingular quasi-projective algebraic variety, the algebraic cycles of codimension at least $i$ account for all the classes of coherent sheaves of codimension at least $i$, up to torsion. More precisely, given an algebraic cycle, each component of it is a subvariety $Z \subseteq X$; the coherent sheaf $\mathcal{O}_{Z}$ on $X$ gives a class $\left[\mathcal{O}_{Z}\right]$ in $K_{0}^{\prime}(X)$. The resulting well-defined map $C H^{i}(X) \rightarrow G r^{i} K_{0}^{\prime}(X)$ induces an isomorphism $C H^{i}(X)_{\mathbb{Q}} \stackrel{\cong}{=} G r^{i} K_{0}^{\prime}(X)_{\mathbb{Q}}[21$, XIV 4.2, IV 2.9]. The proof involves the use of Chern classes to construct an inverse map, and indeed, the Chern character, as defined by Grothendieck, gives the following isomorphism of rings $[13,15.2 .16(\mathrm{~b})]$. 


$$
\operatorname{ch}: K_{0}(X)_{\mathbb{Q}} \cong \bigoplus_{i} C H^{i}(X)_{\mathbb{Q}}
$$

In [37] Quillen defined, for $n \geq 0$, the higher algebraic $K$-group $K_{n}(X)$ for a variety $X$ as the homotopy group $\pi_{n} K(X)$ of a certain topological space $K(X)$ constructed from the category of locally free coherent sheaves on $X$, and he defined $K_{n}^{\prime}(X)$ for a variety $X$ as the homotopy group $\pi_{n} K^{\prime}(X)$ of a certain topological space $K^{\prime}(X)$ constructed in the same way from the category of coherent sheaves on $X$. For a nonsingular quasi-projective algebraic variety $X$, the natural map $K_{n}(X) \rightarrow K_{n}^{\prime}(X)$ is an isomorphism and both groups have filtrations analogous to those for $K_{0}$ and $K_{0}^{\prime}$. It follows from the main result in [16] that the map respects the filtrations in the sense that $F_{\gamma}^{i} K_{n}(X)$ lands in $F^{i-n} K_{n}^{\prime}(X)$ but the filtrations may disagree rationally. We may suspect such behavior by considering the spectrum of a field: it has topological dimension 0 , but its étale cohomological dimension can be greater than 0 , and the higher $K$ groups harbor elements whose Chern characters involve the higher cohomology groups.

An immediate question is whether there is an analogue of (1.1) for the higher $K$-groups that reflects the $\gamma$-filtration on $K_{n}(X)$.

$$
\text { ch : } K_{n}(X)_{\mathbb{Q}} \cong \bigoplus_{i}(?)_{\mathbb{Q}}
$$

The abelian groups replacing the question mark should have an interesting structure in the sense that cognate groups should exist which handle torsion coefficient groups.

\section{Topological K-theory and cohomology}

In the late 1950's Atiyah and Hirzebruch combined Grothendieck's formalism with Bott's periodicity theorem to invent a generalized cohomology theory called topological $K$-theory. In this section we sketch the definition of topological $K$-theory and the relationship between it and singular cohomology provided by the Atiyah-Hirzebruch spectral sequence. The basic objects of study are finite cell complexes, and the spectral sequence arises from the skeletal filtration of a cell complex. We follow the discussion in [18]; see also [3].

Let $X$ be a finite cell complex, and let $\mathbb{C}\left(X^{\text {top }}\right)$ denote the topological ring of continuous functions $X \rightarrow \mathbb{C}$. Although this isn't the way it was originally envisioned, it turns out that there is a way [33] to take the topology of a ring into account when defining the algebraic $K$-groups, yielding the topological $K$ groups $K_{n}\left(X^{\text {top }}\right):=K_{n}\left(\mathbb{C}\left(X^{\text {top }}\right)\right)$. Let $K\left(X^{\text {top }}\right)$ denote the space obtained, so that $K_{n}\left(X^{\text {top }}\right)=\pi_{n} K\left(X^{\text {top }}\right)$. The space $K\left(X^{\text {top }}\right)$ is naturally an infinite loop space, with the deloopings getting more and more connected. 
Let $*$ denote the one point space. Bott computed the homotopy groups of $K\left(*^{\text {top }}\right)$.

$$
\begin{aligned}
K_{2 i}\left(*^{\mathrm{top}}\right) & =\pi_{2 i} K\left(*^{\mathrm{top}}\right)=\mathbb{Z}(i) \\
K_{2 i-1}\left(*^{\mathrm{top}}\right) & =\pi_{2 i-1} K\left(*^{\mathrm{top}}\right)=0
\end{aligned}
$$

We write $\mathbb{Z}(i)$ above to mean simply the group $\mathbb{Z}$; the annotation $(i)$ is there simply to inform us that its elements are destined for the $i$-th stage in the weight filtration. The identification $\mathbb{Z}(1)=K_{2}\left(*^{\text {top }}\right)$ can be decomposed as the following sequence of isomorphisms.

$$
\left.K_{2}\left(*^{\text {top }}\right)=\pi_{2} B G \ell\left(\mathbb{C}^{\text {top }}\right) \cong \pi_{2} B G \ell_{1}\left(\mathbb{C}^{\text {top }}\right)\right) \cong \pi_{1}\left(\mathbb{C}^{\times}\right) \cong \mathbb{Z}(1)
$$

A generator for $\pi_{1}\left(\mathbb{C}^{\times}\right)$gives a generator $\beta$ for $K_{2}\left(*^{\text {top }}\right)$. Bott's theorem includes the additional statement that multiplication by $\beta$ gives a homotopy equivalence of spaces $K\left(*^{\text {top }}\right) \rightarrow \Omega^{2} K\left(*^{\text {top }}\right)$. This homotopy equivalence gives us a non-connected delooping $\Omega^{-2} K\left(*^{\text {top }}\right)$ of $K\left(*^{\text {top }}\right)$, which is $K\left(*^{\text {top }}\right)$ itself. These deloopings can be composed to give deloopings of every order, and hence yields an $\Omega$-spectrum called $B U$ that has $K\left(*^{\text {top }}\right)$ as its underlying infinite loop space $\Omega^{\infty} B U$, and whose homotopy group in dimension $2 i$ is $\mathbb{Z}(i)$, for every integer $i$.

There is a homotopy equivalence of the mapping space $K\left(*^{\text {top }}\right)^{X}$ with $K\left(X^{\text {top }}\right)$ and from this it follows that $K_{n}\left(X^{\text {top }}\right)=\left[X_{+}, \Omega^{\infty} \Omega^{n} B U\right]$, where $X_{+}$denotes $X$ with a disjoint base point adjoined. When $n<0$ there might be a bit of ambiguity about what we might mean when we write $K_{n}\left(X^{\text {top }}\right)$; we let it always denote $\left[X_{+}, \Omega^{\infty} \Omega^{n} B U\right]$, so that $K_{n}\left(X^{\text {top }}\right)=K_{n+2}\left(X^{\text {top }}\right)$ for all $n \in \mathbb{Z}$.

It is a theorem of Atiyah and Hirzebruch that the Chern character for topological vector bundles gives an the following isomorphism.

$$
\operatorname{ch}: K_{n}\left(X^{\mathrm{top}}\right)_{\mathbb{Q}} \cong \bigoplus_{i} H^{2 i-n}(X, \mathbb{Q})
$$

For $n=0$ comparison of this formula with (1.1) shows us that $C H^{i}(X)_{\mathbb{Q}}$ (defined for a variety $X$ ) is a good algebraic analogue of $H^{2 i}(X, \mathbb{Q})$ (defined for a topological space $X$ ).

The isomorphism (2.4) was obtained in [2] from a spectral sequence known as the Atiyah-Hirzebruch spectral sequence. One construction of the spectral sequence uses the skeletal filtration $\operatorname{sk}_{p} X$ of $X$ as follows. (Another one maps $X$ into the terms of the Postnikov tower of $B U$, as we'll see in section 4.)

A cofibration sequence $A \subseteq B \rightarrow B / A$ of pointed spaces and an $\Omega$ spectrum $E$ give rise to a long exact sequence $\cdots \rightarrow\left[A, \Omega^{\infty} \Omega^{1} E\right] \rightarrow$ $\left[B / A, \Omega^{\infty} E\right] \rightarrow\left[B, \Omega^{\infty} E\right] \rightarrow\left[A, \Omega^{\infty} E\right] \rightarrow\left[B / A, \Omega^{\infty} \Omega^{-1} E\right] \rightarrow \cdots$.

We introduce the following groups.

$$
\begin{aligned}
E_{1}^{p q} & :=\left[\operatorname{sk}_{p} X / \operatorname{sk}_{p-1} X, \Omega^{\infty} \Omega^{-p-q} B U\right] \\
D_{1}^{p q} & :=\left[\left(\operatorname{sk}_{p} X\right)_{+}, \Omega^{\infty} \Omega^{-p-q} B U\right]
\end{aligned}
$$


The long exact sequence provides an exact couple $\cdots \rightarrow D_{1}^{p-1, q} \rightarrow E_{1}^{p q} \rightarrow$ $D_{1}^{p q} \rightarrow D_{1}^{p-1, q+1} \rightarrow \ldots$ The explicit computation of the homotopy groups of $B U$ presented above, together with fact that the space $\operatorname{sk}_{p} X / \mathrm{sk}_{p-1} X$ is a bouquet of the $p$-cells from $X$, leads to the computation that

$$
E_{1}^{p q}= \begin{cases}C^{p}(X, \mathbb{Z}(-q / 2)) & \text { if } q \text { is even } \\ 0 & \text { if } q \text { is odd }\end{cases}
$$

where $C^{p}$ denotes the group of cellular cochains. We will abbreviate this conclusion by regarding $\mathbb{Z}(-q / 2)$ as zero when $q$ is odd. The differential $d_{1}: E_{1}^{p q} \rightarrow E_{1}^{p+1, q}$ is seen to be the usual differential for cochains, so that $E_{2}^{p q}=H^{p}(X, \mathbb{Z}(-q / 2))$. The exact couple gives rise to a convergent spectral sequence because $X$ is a finite dimensional cell complex. The abutment is $\left[X_{+}, \Omega^{\infty} \Omega^{-p-q} B U\right]=K_{-p-q}\left(X^{\mathrm{top}}\right)$, so the resulting spectral sequence may be displayed as follows.

$$
E_{2}^{p q}=H^{p}(X, \mathbb{Z}(-q / 2)) \Rightarrow K_{-p-q}\left(X^{\mathrm{top}}\right)
$$

This spectral sequence is concentrated in quadrants I and IV, is nonzero only in the rows where $q$ is even, and is periodic with respect to the translation $(p, q) \mapsto(p, q-2)$. Using the Chern character Atiyah and Hirzebruch show that the differentials in this spectral sequence vanish modulo torsion, and obtain the canonical isomorphism (2.4).

The odd-numbered rows in the spectral sequence (2.8) are zero, so the even-numbered differentials are, also. The spectral sequence can be reindexed to progress at double speed, in which case it will be indexed as follows.

$$
E_{2}^{p q}=H^{p-q}(X, \mathbb{Z}(-q)) \Rightarrow K_{-p-q}\left(X^{\mathrm{top}}\right)
$$

\section{The motivic spectral sequence}

Now let $X$ be a nonsingular algebraic variety, or more generally, a regular scheme.

Consider a finitely generated regular ring $A$ and a prime number $\ell$. In [38], motivated by the evident success of étale cohomology as an algebraic analogue for varieties of singular cohomology with finite coefficients and by conjectures of Lichtenbaum relating $K$-theory of number rings to étale cohomology, Quillen asked whether there is a spectral sequence analogous to $(2.8)$ of the following form, converging at least in degrees $-p-q>\operatorname{dim}(A)+1$.

$$
E_{2}^{p q}=H_{e t}^{p}\left(\operatorname{Spec}\left(A\left[\ell^{-1}\right]\right), \mathbb{Z}_{\ell}(-q / 2)\right) \Rightarrow K_{-p-q}(A) \otimes \mathbb{Z}_{\ell}
$$

The spectral sequence would degenerate in case $A$ is the ring of integers in a number field and either $\ell$ is odd or $A$ is totally imaginary. 
Beilinson asked ([4], see also [5, p. 182]) whether there is an integral version of (3.1) serving as a totally algebraic analogue of (2.8) that would look like this:

$$
E_{2}^{p q}=H^{p}(X, \mathbb{Z}(-q / 2)) \Rightarrow K_{-p-q}(X)
$$

The groups $H^{p}(X, \mathbb{Z}(-q / 2))$ would be called motivic cohomology groups, and comparison with (1.1) suggests we demand that $H^{2 i}(X, \mathbb{Z}(i))=C H^{i}(X)$. In Beilinson's formulation, $\mathbb{Z}(i)$ would be a cohomological complex of sheaves of abelian groups in the Zariski topology on $X$ concentrated in degrees $1,2, \ldots, t$ (except for $t=0$, where $\mathbb{Z}(0)=\mathbb{Z})$ and $H^{p}(X, \mathbb{Z}(-q / 2)$ ) would be the hypercohomology of the complex. In an alternative formulation [30] advanced by Lichtenbaum the complex $\mathbb{Z}(i)$ is derived from such a complex in the étale topology on $X$.

An alternative indexing scheme for the spectral sequence eliminates the odd-numbered rows whose groups are zero anyway, and looks like this.

$$
E_{2}^{p q}=H^{p-q}(X, \mathbb{Z}(-q)) \Rightarrow K_{-p-q}(X)
$$

\section{Filtrations as a source of spectral sequences}

The most basic way to make a (convergent) spectral sequence is to start with a homological bicomplex [31, XI.6], but for more generality one can also start with a filtered chain complex [31, XI.3], or even just with an exact couple [31, XI.5]. An exact couple is basically an exact triangle of bigraded abelian groups, where two of the three terms are the same, as in the following diagram.

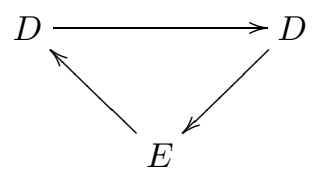

Such exact couples can arise from long exact sequences where the terms are index by a pair of integers, and, aside from a difference of indices, two of the terms look the same. If $C$ is a chain complex, and $C=F^{0} C \supseteq F^{1} C \supseteq F^{2} C \supseteq$ ... is a descending filtration by subcomplexes, then the long exact sequences

$$
\cdots \rightarrow H_{q}\left(F^{p+1} C\right) \rightarrow H_{q}\left(F^{p} C\right) \rightarrow H_{q}\left(F^{p} C / F^{p+1} C\right) \rightarrow H_{q-1}\left(F^{p+1} C\right) \rightarrow \ldots
$$

provide the exact couple that in turn provides the spectral sequence associated to the filtration.

More generally, we could start simply with a sequence of maps $C=$ $F^{0} C \leftarrow F^{1} C \leftarrow F^{2} C \leftarrow \ldots$, for we may replace the quotient chain complex $F^{p} C / F^{p+1} C$ with the mapping cone of the map $F^{p} C \leftarrow F^{p+1} C$, preserving the basic shape of the long exact sequences above, which is all that is needed to make an exact couple. 
Another source of long exact sequences is homotopy theory, where a fibration sequence $F \rightarrow X \rightarrow Y$ of pointed spaces gives rise to a long exact sequence $\cdots \rightarrow \pi_{n} F \rightarrow \pi_{n} X \rightarrow \pi_{n} Y \rightarrow \pi_{n-1} F \rightarrow \cdots \rightarrow \pi_{0} X \rightarrow \pi_{0} Y$. If $X \rightarrow Y$ is any map of pointed spaces, then letting $F$ be the homotopy fiber of the map provides the desired fibration sequence. If we insist that $X$ and $Y$ be homotopy commutative group-like $H$-spaces (Abelian groups up to homotopy) and that the map $X \rightarrow Y$ be compatible with the $H$-space structure, then $F$ will be an $H$-space, too, the terms in the long exact sequence will be abelian groups, and the maps in it will be homomorphisms. Finally, if we assume that $\pi_{0} X \rightarrow \pi_{0} Y$ is surjective, and define the $\pi_{n}=0$ for $n<0$, the long exact sequence will be exact also at $\pi_{0} Y$ and thus will extend infinitely far in both directions. Ultimately, we may assume that $X \rightarrow Y$ is a map of spectra, in which case a homotopy cofiber for the map $X \rightarrow Y$ exists, serving as a complete analogue of the mapping cone for a map of chain complexes.

The long exact sequences of homotopy theory can produce spectral sequences, too. For example, let $Y$ be a connected space with abelian fundamental group, and let $Y=F^{0} Y \leftarrow F^{1} Y \leftarrow F^{2} Y \leftarrow \ldots$ be a sequence of maps between connected spaces $F^{p} Y$ with abelian fundamental group; call such a thing a filtration of $Y$ or also a tower. By a slight abuse of notation let $\Omega F^{p / p+1} Y$ denote the homotopy fiber of the map $F^{p+1} Y \rightarrow F^{p} Y$. The long exact sequences $\cdots \rightarrow \pi_{q} F^{p+1} Y \rightarrow \pi_{q} F^{p} Y \rightarrow \pi_{q-1} \Omega F^{p / p+1} Y \rightarrow \pi_{q-1} F^{p+1} Y \rightarrow \ldots$ form an exact couple. (Observe also that $\pi_{0} \Omega F^{p / p+1} Y$ appears in the long exact sequence as the cokernel of a homomorphism between abelian groups, so naturally is one as well.) The corresponding spectral sequence will converge to $\pi_{n} Y$ if for every $q$ and for every sufficiently large $p, \pi_{q} F^{p} Y=0$ [12, A.6].

If $Y$ is a space, then the Postnikov tower of $Y$ is a filtration $Y=F^{0} Y \leftarrow$ $F^{1} Y \leftarrow F^{2} Y \leftarrow \ldots$ that comes equipped with spaces $F^{p / p+1} Y$ fitting into fibration sequences $F^{p+1} Y \rightarrow F^{p} Y \rightarrow F^{p / p+1} Y$, and $F^{p / p+1} Y$ is an Eilenberg MacLane space $K\left(\pi_{p} Y, p\right)$. The corresponding spectral sequence is uninteresting, because it gives no new information about the homotopy groups $\pi_{p} Y$ or the space $Y$.

More generally, let $Y$ be a spectrum, let $Y=F^{0} Y \leftarrow F^{1} Y \leftarrow F^{2} Y \leftarrow \ldots$ is a sequence of maps of spectra; call such a thing a filtration of $Y$. Let $F^{p / p+1} Y$ denote the homotopy cofiber of the map $F^{p+1} Y \rightarrow F^{p} Y$; we call it the $p$-th layer of the filtration. The long exact sequences $\cdots \rightarrow \pi_{q} F^{p+1} Y \rightarrow$ $\pi_{q} F^{p} Y \rightarrow \pi_{q} F^{p / p+1} Y \rightarrow \pi_{q-1} F^{p+1} Y \rightarrow \ldots$ form an exact couple. The corresponding spectral sequence will converge to $\pi_{n} Y$ if for every $q$ and for every sufficiently large $p, \pi_{q} F^{p} Y=0$.

If $Y$ is a spectrum, possibly with negative homotopy groups, then the Postnikov filtration has terms $F^{p} Y$ with $p<0$.

The Postnikov filtration of the spectrum $B U$ involves Eilenberg-MacLane spaces $F^{2 i / 2 i+1} B U \cong K(\mathbb{Z}, 2 i)$; the other steps in the filtration are trivial. Taking a finite cell complex $X$, the mapping spectra $\left(F^{p} B U\right)^{X}$ provide a filtration of $Y:=B U^{X} \cong K\left(X^{\text {top }}\right)$ and fit into fibration sequences $\left(F^{2 i+1} B U\right)^{X} \rightarrow$ $\left(F^{2 i} B U\right)^{X} \rightarrow K(\mathbb{Z}, 2 i)^{X}$. The homotopy groups of $K(\mathbb{Z}, 2 i)^{X}$ turn out to 
be cohomology groups of $X$, for $\pi_{p} K(\mathbb{Z}, 2 i)^{X} \cong\left[X_{+} \wedge S^{p}, K(\mathbb{Z}, 2 i)\right] \cong$ $\left[X_{+}, \Omega^{p} K(\mathbb{Z}, 2 i)\right] \cong\left[X_{+}, K(\mathbb{Z}, 2 i-p)\right] \cong H^{2 i-p}(X, \mathbb{Z})$. Indeed, the spectrum $K(\mathbb{Z}, 2 i)^{X}$ is the generalized Eilenberg-MacLane spectrum corresponding to the singular cochain complex of $X$, shifted in degree by $2 i$ [10, IV.2.4-5], and the spectral sequence resulting from this filtration can be identified with the Atiyah-Hirzebruch spectral sequence of $X$, defined as above using the skeletal filtration of $X$; see [20, Theorem B.8].

In general, given a filtration of a spectrum $Y$, we may ask that the layers $F^{p / p+1} Y$ be generalized Eilenberg-MacLane spectra, i.e., should come from chain complexes of abelian groups. Intuitively, such a spectral sequence describes something complicated (homotopy groups) in terms of something simpler and hopefully more computable (homology or cohomology groups). Ideally, those chain complexes would be explicitly constructible without using any higher homotopy groups.

The motivic spectral sequences turn out to be of the type just described, and it's not surprising, because constructing a filtration of a spectrum is a natural way to proceed, postponing as long as possible the study of the homotopy groups themselves. That is the hope expressed in [18].

Here is a further important motivational remark of Goodwillie. If $R$ is a commutative ring and $Y$ is the $K$-theory spectrum $K(R)$ derived from projective finitely generated $R$-modules, then tensor product over $R$ makes $Y$ into a ring spectrum. If the proposed filtration is compatible with products, then $F^{0 / 1} Y$ is also a ring spectrum and each cofiber $F^{p / p+1} Y$ is a module over it. If, moreover, $F^{0 / 1} Y$ is the Eilenberg-MacLane spectrum $H_{\mathbb{Z}}$ that classifies ordinary homology, then it follows that $F^{p / p+1} Y$ must be an Eilenberg-MacLane spectrum, too, i.e., come from a chain complex of abelian groups. Further work may be required to make the chain complex explicit, but at least our search for a suitable filtration can be limited to those compatible with products.

\section{Commuting automorphisms}

In this section we explain the Goodwillie-Lichtenbaum idea involving tuples of commuting automorphisms and the theorem [19] that gives a spectral sequence relating $\mathrm{K}$-theory to chain complexes constructed from direct-sum Grothendieck groups of tuples of commuting automorphisms. (The intrusion of of the direct-sum Grothendieck groups during the construction of the spectral sequence was unwelcome.) One aspect of the proof I want to emphasize is the "cancellation" theorem for the $\operatorname{space} \operatorname{Stab}(P, Q)$ of stable isomorphisms between projective modules $P$ and $Q$. It says that $\operatorname{Stab}(P, Q)$ and $\operatorname{Stab}(P \oplus X, Q \oplus X)$ are homotopy equivalent, and requires the ground ring to be a connected simplicial ring. The proof is sort of similar to Voevodsky's proof of his cancellation theorem, which we'll cover in section 6 . 
Let's consider an affine regular noetherian scheme $X$. The Fundamental Theorem [37] says that the map $K(X) \rightarrow K\left(X \times \mathbb{A}^{1}\right)$ is a homotopy equivalence; this property of the functor $K$ is called homotopy invariance.

There is a standard way of converting a functor into one that satisfies homotopy invariance, first used ${ }^{2}$ by Gersten in [15] to describe the higher $K$-theory of rings developed by Karoubi and Villamayor in [23], an attempt which turned out to give the right answer for regular noetherian commutative rings. The standard topological simplices $\Delta^{n}$ form a cosimplicial space $\Delta^{*}$ : $n \mapsto \Delta^{n}$ in which the transition maps are affine maps that send vertices to vertices. For example, some of the transition maps are the inclusion maps $\Delta^{n-1} \hookrightarrow \Delta^{n}$ whose images are the faces of codimension 1 . Gersten considered the analogous cosimplicial affine space $\mathbb{A}^{\cdot}: n \mapsto \mathbb{A}^{n}$ whose transition maps are given by the same formulas. We regard $\mathbb{A}^{n}$ as a simplex, the set of affine linear combinations of $n+1$ distinguished points which are called its vertices; its faces are the subaffine spaces spanned by subsets of the vertices. It is an elementary fact (see, for example, [19]) that if $F$ is a contravariant functor to spaces from a category of smooth varieties that includes the affine spaces, the $G(X):=\left|n \mapsto F\left(X \times \mathbb{A}^{n}\right)\right|$ is homotopy invariant, and the map $F \rightarrow G$ is, in some up-to-homotopy sense, the universal map to a homotopy invariant functor.

Let $K\left(X \times \mathbb{A}^{\cdot}\right)$ denote the geometric realization of simplicial space $n \mapsto$ $K\left(X \times \mathbb{A}^{n}\right)$. It follows that the map $K(X) \rightarrow K\left(X \times \mathbb{A}^{*}\right)$ is a homotopy equivalence. The simplicial space $n \mapsto K\left(X \times \mathbb{A}^{n}\right)$ is analogous to a bicomplex, with the spaces $K\left(X \times \mathbb{A}^{n}\right)$ playing the role of the columns. This particular simplicial space isn't interesting, because the face and degeneracy maps are homotopy equivalences, but we can relate it to some simplicial spaces that are.

Suppose $X$ and $Y$ are separated noetherian schemes. Then the union of two subschemes of $X \times Y$ that are finite over $X$ will also be finite over $X$, and thus an extension of two coherent sheaves on $X \times Y$ whose supports are finite over $X$ will also be finite over $X$. Thus we may define the exact category $\mathcal{P}(X, Y)$ consisting of those coherent sheaves on $X \times Y$ that are flat over $X$ and whose support is finite over $X$. If $X^{\prime} \rightarrow X$ is a map, then there is an exact base-change functor $\mathcal{P}(X, Y) \rightarrow \mathcal{P}\left(X^{\prime}, Y\right)$.

For example, the category $\mathcal{P}(X, \operatorname{Spec} \mathbb{Z})$ is equivalent to the category $\mathcal{P}(X)$ of locally free coherent sheaves on $X$. When $X=\operatorname{Spec}(R)$ is affine, $\mathcal{P}(X)$ is equivalent to the category $\mathcal{P}(R)$ of finitely generated projective $R$-modules. When $X=\operatorname{Spec}(R)$ and $Y=\operatorname{Spec}(S)$ are both affine, the category $\mathcal{P}(X, Y)$ is equivalent to the category $\mathcal{P}(R, S)$ of $R$-S-bimodules that are finitely generated and projective as $R$-modules.

The category $\mathcal{P}\left(X, \mathbb{A}^{1}\right)$ is equivalent to the category of pairs $(M, f)$ where $M$ is a locally free coherent sheaf on $X$ and $f$ is an endomorphism of $M$. If $R$ is a commutative ring and $X$ is the affine scheme $\operatorname{Spec} R$, then $M$ is essentially

\footnotetext{
${ }^{2}$ according to $[1$, p. 78$]$
} 
the same as a finitely generated projective $R$-module. Similarly, the category $\mathcal{P}\left(X, \mathbb{A}^{n}\right)$ is equivalent to the category of tuples $\left(M, f_{1}, \ldots, f_{n}\right)$ where $M$ is a locally free coherent sheaf on $X$ and $f_{1}, \ldots, f_{n}$ are commuting endomorphisms of $M$.

Let $\mathbb{G}_{m}=\operatorname{Spec} \mathbb{Z}\left[u, u^{-1}\right]$. (It is a group scheme, but we won't use its multiplication operation.) The category $\mathcal{P}\left(X, \mathbb{G}_{m}\right)$ is equivalent to the category of pairs $(M, f)$ where $M$ is a locally free coherent sheaf on $X$ and $f$ is an automorphism of $M$. The category $\mathcal{P}\left(X, \mathbb{G}_{m}^{n}\right)$ is equivalent to the category of tuples $\left(M, f_{1}, \ldots, f_{n}\right)$ where $M$ is a locally free coherent sheaf on $X$ and $f_{1}, \ldots, f_{n}$ are commuting automorphisms of $M$.

The identity section $*:$ Spec $\mathbb{Z} \rightarrow \mathbb{G}_{m}$ of $\mathbb{G}_{m}$ plays the role of the base point, so let $*$ denote it. Following Voevodsky, more or less, define the algebraic circle $\mathbb{S}^{1}$ to be the pair $\left(\mathbb{G}_{m}, *\right)$. Consider the following commutative diagram.

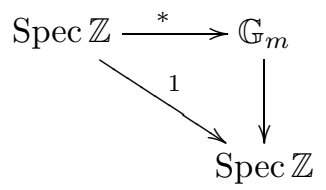

From it we see that if $F$ is any contravariant (or covariant) functor from schemes to abelian groups, we can define $F\left(\mathbb{S}^{1}\right)$ as the complementary summand in the decomposition $F\left(\mathbb{G}_{m}\right) \cong F(\operatorname{Spec} \mathbb{Z}) \oplus F\left(\mathbb{S}^{1}\right)$ derived from the following diagram.

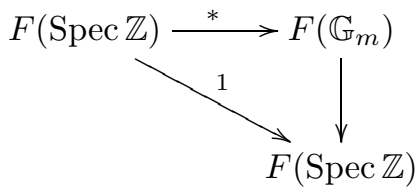

The result will be natural in $F$. We can iterate this: considering $F(X \times Y)$ first as a functor of $X$ we can give a meaning to $F\left(\mathbb{S}^{1} \times Y\right)$, and then considering $F\left(\mathbb{S}^{1} \times Y\right)$ as a functor of $Y$ we can give a meaning to $F\left(\mathbb{S}^{1} \times \mathbb{S}^{1}\right)$. Usually that would be written as $F\left(\mathbb{S}^{1} \wedge \mathbb{S}^{1}\right)$ if $\mathbb{S}^{1}$ is regarded as a pointed object rather than as a pair. We may also define $\mathbb{S}^{t}:=\mathbb{S}^{1} \times \cdots \times \mathbb{S}^{1}$ as a "product" of $t$ copies of $\mathbb{S}^{1}$, and then $F\left(\mathbb{S}^{t}\right)$, interpreted as above, is the summand of $F\left(\mathbb{G}_{m}^{t}\right)$ that is new in the sense that it doesn't come from $F\left(\mathbb{G}_{m}^{t-1}\right)$ via any of the standard inclusions.

We define $\mathbb{Z}^{\operatorname{ex}}(t)(X)$ to be the chain complex associated to the simplicial abelian group $n \mapsto K_{0}\left(\mathcal{P}\left(X \times \mathbb{A}^{n}, \mathbb{S}^{t}\right)\right)$, regarded as a cohomological chain complex, and shifted so that the group $K_{0}\left(\mathcal{P}\left(X \times \mathbb{A}^{t}, \mathbb{S}^{t}\right)\right)$ is in degree 0 . In a context where a complex $\mathbb{Z}^{\text {ex }}(t)$ of sheaves is required, we sheafify the presheaf $U \mapsto \mathbb{Z}^{\operatorname{ex}}(t)(U)$, where $U$ ranges over open subsets of $X$.

Recall the direct sum Grothendieck group $K_{0}^{\oplus}(\mathcal{M})$, where $\mathcal{M}$ is a small additive category. It is defined to be the abelian group given by generators $[M]$, one for each object $M$ of $\mathcal{M}$, and by relations $[M]=\left[M^{\prime}\right]+\left[M^{\prime \prime}\right]$, one for each isomorphism of the form $M \cong M^{\prime} \oplus M^{\prime \prime}$. If $\mathcal{M}$ is not small, but 
equivalent to an additive category $\mathcal{M}^{\prime}$ that is, we define $K_{0}^{\oplus}(\mathcal{M}):=K_{0}^{\oplus}\left(\mathcal{M}^{\prime}\right)$ and observe that up to isomorphism it is independent of the choice of $\mathcal{M}^{\prime}$. It is an easy exercise to check that two classes $[M]$ and $[N]$ are equal in $K_{0}^{\oplus}(\mathcal{M})$ if and only if $M$ and $N$ are stably isomorphic, i.e., there is another object $C$ such that $M \oplus C \cong N \oplus C$. There is an evident natural surjection $K_{0}^{\oplus}(\mathcal{M}) \rightarrow K_{0}(\mathcal{M})$.

Now we present the motivic cohomology complex encountered in [19]. We define $\mathbb{Z}^{\oplus}(t)(X)$ to be the chain complex associated to the simplicial abelian group $n \mapsto K_{0}^{\oplus}\left(\mathcal{P}\left(X \times \mathbb{A}^{n}, \mathbb{S}^{t}\right)\right)$, reversed and shifted as for $\mathbb{Z}^{\operatorname{ex}}(t)(X)$, and we define a complex of sheaves $\mathbb{Z}^{\oplus}(t)$ as before. There is a natural map $\mathbb{Z}^{\oplus}(t) \rightarrow$ $\mathbb{Z}^{\operatorname{ex}}(t)$.

If $X=\operatorname{Spec}(R)$ is the spectrum of a regular noetherian $\operatorname{ring} R$, then from $[19,9.7]$ one derives a spectral sequence of the following form.

$$
E_{2}^{p q}=H^{p-q}\left(\mathbb{Z}^{\oplus}(-q)(X)\right) \Rightarrow K_{-p-q}(X)
$$

The rest of this section will be devoted to sketching some of the details of the proof.

The coordinate rings $n \mapsto R\left[\mathbb{A}^{n}\right]$ form a contractible simplicial ring, where $R\left[\mathbb{A}^{n}\right]=R\left[T_{1}, \ldots, T_{n}\right]$. Let's examine the part in degrees 0 and 1 , where we have the ring homomorphism $R \hookrightarrow R[T]$ and the evaluation maps $e_{0}, e_{1}$ : $R[T] \rightrightarrows R$ defined by $f \mapsto f(0)$ and $f \mapsto f(1)$. The two evaluation maps allow us to regard a polynomial in $R[T]$, a matrix over $R[T]$, or an $R[T]$ module, as a sort of homotopy connecting the two specializations obtained using the two evaluation maps. There are two simple remarks about such algebraic homotopies that play a role in the proofs.

Remark 5.1. Firstly, short exact sequences always split up to homotopy, so working with direct sum $K$-theory might not be so bad. Here is the homotopy. Start with a short exact sequence $E: 0 \rightarrow M^{\prime} \rightarrow M \rightarrow M^{\prime \prime} \rightarrow 0$ of $R$-modules, and define an $R[T]$-module $\tilde{M}$ as the pull back in the following diagram.

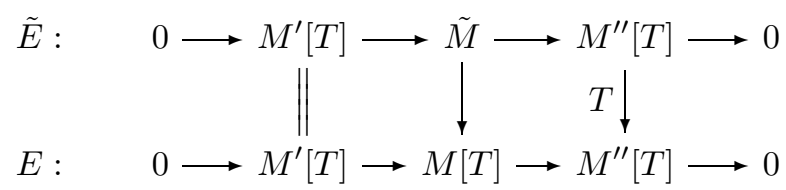

The short exact sequence $\tilde{E}$ specializes to $E$ when $T=1$ and to $0 \rightarrow M^{\prime} \rightarrow$ $M^{\prime} \oplus M^{\prime \prime} \rightarrow M^{\prime \prime} \rightarrow 0$ when $T=0$, and provides the desired homotopy.

Remark 5.2. Secondly, signed permutation matrices of determinant 1 don't matter, as they are homotopic to the identity. Here is an example of such a homotopy: the invertible matrix

$$
\left(\begin{array}{cc}
1 & -T \\
0 & 1
\end{array}\right)\left(\begin{array}{ll}
1 & 0 \\
T & 1
\end{array}\right)\left(\begin{array}{cc}
1 & -T \\
0 & 1
\end{array}\right)
$$

specializes to $\left(\begin{array}{ll}1 & 0 \\ 0 & 1\end{array}\right)$ at $T=0$ and to $\left(\begin{array}{cc}0 & -1 \\ 1 & 0\end{array}\right)$ at $T=1$. 
Here is an application of the second remark that played a role in the proof in [19], abstracted for examination. Suppose $\mathcal{M}$ is an additive category. Given two objects $M$ and $N$ of $\mathcal{M}$ let's define a new category $\operatorname{Stab}(M, N)$, the category of stable equivalences between $M$ and $N$. A stable equivalence is a pair $(C, \theta)$ where $C \in \mathcal{M}$ and $\theta: M \oplus C \stackrel{\cong}{\rightarrow} N \oplus C$. An object of $\operatorname{Stab}(M, N)$ is a stable equivalence. An arrow $(C, \theta) \rightarrow\left(C^{\prime}, \theta^{\prime}\right)$ of $\operatorname{Stab}(M, N)$ is an isomorphism class of pairs $(D, \psi)$ with $D \in \mathcal{M}$ and $\psi: C \oplus D \stackrel{\cong}{\rightrightarrows} C^{\prime}$, such that $\theta^{\prime}=\left(1_{N} \oplus \psi\right)\left(\theta \oplus 1_{D}\right)\left(1_{M} \oplus \psi\right)^{-1}$. In effect, an arrow connects a stable isomorphism to one obtained from it by direct sum with an identity isomorphism, underscoring the point that stable isomorphisms related in that way are not much different from each other.

Given $P \in \mathcal{M}$ there are natural functors $\operatorname{Stab}(M, N) \leftrightarrows \operatorname{Stab}(M \oplus P, N \oplus$ $P)$. The rightward map $\mu$ adds the identity isomorphism $1_{P}$ to a stable isomorphism of $M$ with $N$, getting a stable isomorphism of $M \oplus P$ with $N \oplus P$. The leftward map $\rho$ sends an object $(D, \psi)$ to $(P \oplus D, \psi)$.

The composite functor $\operatorname{Stab}(M, N) \stackrel{\mu}{\longrightarrow} \operatorname{Stab}(M \oplus P, N \oplus P) \stackrel{\rho}{\rightarrow} \operatorname{Stab}(M, N)$ is the target of a natural transformation whose source is the identity functor. The arrows in the transformation connect $(C, \theta)$ to something equivalent to $\left(C \oplus P, \theta \oplus 1_{P}\right)$. After geometric realization the natural transformation gives a homotopy from the identity to $\rho \circ \mu$.

Our goal is to show the functors $\rho$ and $\mu$ are inverse homotopy equivalences. It turns out there is a switching swindle that produces a homotopy from $\mu \circ \rho \sim 1$ for free from the homotopy $1 \sim \rho \circ \mu$, which we describe now.

The composite functor $\operatorname{Stab}(M \oplus P, N \oplus P) \stackrel{\rho}{\rightarrow} \operatorname{Stab}(M, N) \stackrel{\mu}{\rightarrow} \operatorname{Stab}(M \oplus$ $P, N \oplus P)$ sends an object $(D, \psi)$, where $\psi:(M \oplus P) \oplus D \stackrel{\cong}{\longrightarrow}(N \oplus P) \oplus D$, to an object of the form $(P \oplus D, \beta)$. The isomorphism $\beta:(M \oplus P) \oplus P \oplus D \cong$ $(N \oplus P) \oplus P \oplus D$ has $1_{P}$ as a direct summand, provided by $\mu$, but the identity map is on the first $P$, which is the wrong one! If only it were on the second one, we could get a natural transformation as before. An equivalent way to visualize the situation is to embed the composite map into the following diagram.

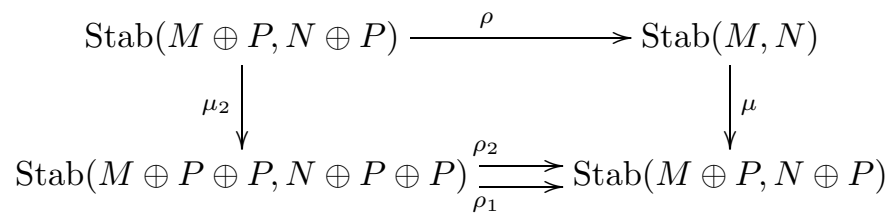

Here $\rho_{1}$ and $\rho_{2}$ are analogues of $\rho$ that deal with the first and second $P$, respectively, and $\mu_{2}$ adds in an identity map on the second $P$. The square commutes up to homotopy if $\rho_{2}$ is used, so we need a homotopy $\rho_{1} \sim \rho_{2}$. The trick used is to put ourselves in a world where remark 5.2 applies and incorporate a homotopy from the identity map to a signed permutation that switches one copy of $P$ with the other. That world is the world where we are working over the simplicial coordinate ring $R\left[\mathbb{A}^{\cdot}\right]$ of $\mathbb{A}^{\cdot}$, i.e., $\mathcal{M}$ is replaced 
by a simplicial additive category $n \mapsto \mathcal{M}_{n}$ over $R\left[\mathbb{A}^{\cdot}\right]$, so such homotopies are available. The details of that homotopy are the main technical point of $[19,8.3]$. Following Voevodsky, we may refer to the homotopy equivalence $\operatorname{Stab}(M, N) \sim \operatorname{Stab}(M \oplus P, N \oplus P)$ as a cancellation theorem. Now we explain briefly how it gets used in the construction of the spectral sequence (5.1).

We recall $S^{-1} S(\mathcal{M})$, the category constructed by Quillen in [17] whose homotopy groups are the higher direct sum $K$-groups of the additive category $\mathcal{M}$. Its objects are pairs $(M, N)$ of objects in $\mathcal{M}$, and an arrow $(M, N) \rightarrow$ $\left(M^{\prime}, N^{\prime}\right)$ is an isomorphism class of triples $(C, \alpha, \beta)$ where $C \in \mathcal{M}$ and $\alpha$ : $M \oplus C \stackrel{\cong}{\longrightarrow} M^{\prime}$ and $\beta: N \oplus C \stackrel{\cong}{\longrightarrow} N^{\prime}$ are isomorphisms. The construction is designed so $\pi_{0} S^{-1} S(\mathcal{M}) \cong K_{0}^{\oplus}(\mathcal{M})$.

By techniques due to Quillen [37, Theorem B] homotopy equivalences between naive approximations to the homotopy fibers of a functor yield homotopy fibration sequences. The categories $\operatorname{Stab}(M, N)$ appear as naive approximations to the homotopy fibers of the functor from the (contractible) path space of $S^{-1} S(\mathcal{M})$ to $S^{-1} S(\mathcal{M})$. That follows from two observations: a stable isomorphism of $M$ with $N$ is essentially a diagram of the following type in $S^{-1} S(\mathcal{M})$

$$
(0,0) \longrightarrow(M \oplus C, N \oplus C) \longleftarrow(M, N)
$$

and an arrow in the category $\operatorname{Stab}(M, N)$ is essentially a diagram of the following type in $S^{-1} S(\mathcal{M})$.

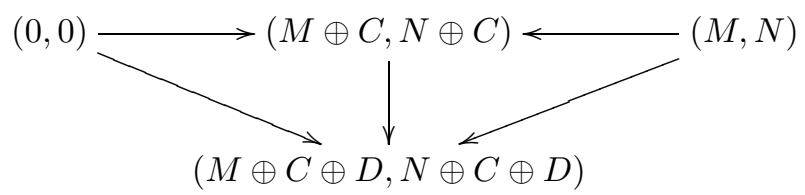

Since all the fibers are the same up to homotopy equivalence, a variant of Quillen's Theorem B adapted to the simplicial world tells us that any of the fibers, say $\operatorname{Stab}(0,0)$, is almost the loop space of $S^{-1} S(\mathcal{M})$. We have to say "almost" because not every object $(M, N)$ of $S^{-1} S(\mathcal{M})$ has components $M$ and $N$ that are stably isomorphic. For an additive category $\mathcal{M}$ the obstruction to stable isomorphism is captured precisely by the group $K_{0}^{\oplus}(\mathcal{M})$. In our situation, $\mathcal{M}$ is a simplicial additive category, which means that the space associated to the simplicial abelian group $n \mapsto K_{0}^{\oplus}\left(\mathcal{M}_{n}\right)$ enters into a fibration with the other two spaces. An object of $\operatorname{Stab}(0,0)$ is a stable isomorphism of 0 with 0 , and is essentially a pair $(C, \theta)$ where $C$ is an object of $\mathcal{M}$ and $\theta$ is an automorphism of $C$. That's how automorphisms enter into the picture. Proceeding inductively, one encounters the additive category whose objects are such pairs $(C, \theta)$. An automorphism of such an object is an automorphism of $C$ that commutes with $\theta$. That explains why, at subsequent stages, tuples of commuting automorphisms are involved. Starting the game off with $\mathcal{M}:=$ $\left(n \mapsto \mathcal{P}\left(R\left[\mathbb{A}^{n}\right]\right)\right)$ one can see now how $\mathbb{Z}^{\oplus}(t)(X)$ arises. 
We discuss the extent to which the map $\mathbb{Z}^{\oplus}(t)(X) \rightarrow \mathbb{Z}(t)(X)$ is a quasiisomorphism in section 6 .

\section{Cancellation and comparison with motivic cohomology}

In this section we describe the cancellation theorem of Voevodsky [45] in the form presented by Suslin in [41, §4]. It is one of the tools used by Suslin to prove that $\mathbb{Z}^{\oplus}(t)(X) \rightarrow \mathbb{Z}(t)(X)$ is a quasi-isomorphism locally on a smooth variety $X$, and we'll also discuss how that goes. We take some liberties with Suslin's presentation for the sake of motivation and for the sake of hiding technicalities. In this section $X=\operatorname{Spec}(R)$ and $Y=\operatorname{Spec}(S)$ are affine regular noetherian schemes.

There is a functor $\mathcal{P}(X, Y) \rightarrow \mathcal{P}\left(X \times \mathbb{G}_{m}, Y \times \mathbb{G}_{m}\right)$ arising from tensor product with the structure sheaf of the graph of the identity map $\mathbb{G}_{m} \rightarrow \mathbb{G}_{m}$.

Let $K_{0}^{\oplus}\left(\mathcal{P}\left(X \times \mathbb{A}^{*}, Y\right)\right)$ denote the simplicial abelian group $n \mapsto K_{0}^{\oplus}(\mathcal{P}(X \times$ $\left.\mathbb{A}^{n}, Y\right)$ ), or when needed, the chain complex associated to it. The cancellation theorem states that the induced map

$$
K_{0}^{\oplus}\left(\mathcal{P}\left(X \times \mathbb{A}^{\dagger}, Y\right)\right) \rightarrow K_{0}^{\oplus}\left(\mathcal{P}\left(X \times \mathbb{S}^{1} \times \mathbb{A}^{i}, Y \times \mathbb{S}^{1}\right)\right)
$$

is a quasi-isomorphism. Roughly speaking, the idea is to use the same switching swindle as in the previous section. One could imagine trying to construct the following diagram, analogous to $(5.2)$, with $\mathbb{S}^{1}$ here playing the role $P$ played there.

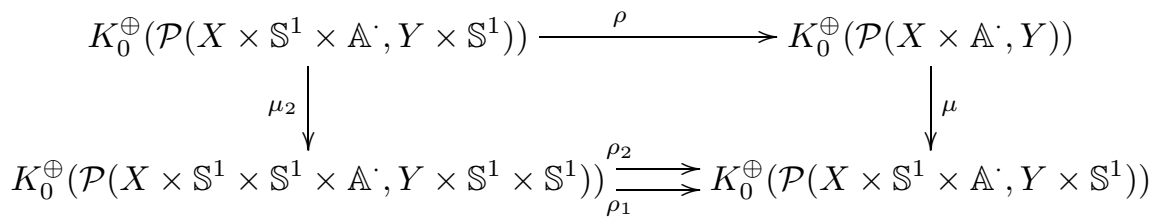

Here the map $\mu$ would be external product with the identity map on $\mathbb{S}^{1}$, and hopefully, one could find a map $\rho$ for which $\rho \circ \mu \sim 1$. The maps $\rho_{1}$ and $\rho_{2}$ would be instances of $\rho$, but based on different $\mathbb{S}^{1}$ factors. Then one could hope to use the switching swindle to get the homotopy $\mu \circ \rho \sim 1$ for free.

We may regard a class $[M]$ of $K_{0}^{\oplus}(\mathcal{P}(X, Y))$ as a direct sum Grothendieck group correspondence from $X$ to $Y$. Intuitively, a correspondence is a relation that relates some points of $Y$ to each point of $X$. Geometrically, we imagine that the points $(x, y)$ in the support of $M$ lying over a point $x \in X$ give the values $y$ of the correspondence. Since the support of $M$ is finite over $X$, each $x$ corresponds to only finitely many $y$. Since $M$ is flat over $X$, the number of points $y$ corresponding to $x$, counted with multiplicity, is the rank of rank of $M$ as a locally free $\mathcal{O}_{X}$-module near $x$, and is a locally constant function of $x$. 
For example, an element of $K_{0}^{\oplus}\left(\mathcal{P}\left(X, \mathbb{A}^{1}\right)\right)$ is essentially a square matrix $\theta$ of regular functions on $X$, and to a point $x$ it associates the eigenvalues of $\theta(x)$. An element of $K_{0}^{\oplus}\left(\mathcal{P}\left(X, \mathbb{G}_{m}\right)\right)$ is essentially an invertible square matrix $\theta$ of regular functions on $X$, and to a point $x$ it associates the eigenvalues of $\theta(x)$, which are nonzero numbers.

We define the additive category of correspondences; its objects are the symbols $[X]$, one for each noetherian separated scheme $X$. An arrow $[X] \rightarrow[Y]$ is an element of $\operatorname{Hom}([X],[Y]):=K_{0}^{\oplus}(\mathcal{P}(X, Y))$. The composition $[N] \circ[M]$ of correspondences is defined to be $\left[M \otimes \mathcal{O}_{Y} N\right]$. The direct sum $[X] \oplus\left[X^{\prime}\right]$ of two objects is represented by the disjoint union $\left[X \sqcup X^{\prime}\right]$ of schemes, because $K_{0}^{\oplus}\left(\mathcal{P}\left(X \sqcup X^{\prime}, Y\right)\right) \cong K_{0}^{\oplus}(\mathcal{P}(X, Y)) \oplus K_{0}^{\oplus}\left(\mathcal{P}\left(X^{\prime}, Y\right)\right)$ and $K_{0}^{\oplus}\left(\mathcal{P}\left(X, Y \sqcup Y^{\prime}\right)\right) \cong$ $K_{0}^{\oplus}(\mathcal{P}(X, Y)) \oplus K_{0}^{\oplus}\left(\mathcal{P}\left(X, Y^{\prime}\right)\right)$. We define $[X] \otimes\left[X^{\prime}\right]:=\left[X \times X^{\prime}\right]$ on objects, and extend it to a bilinear function on arrows.

There is a function $\operatorname{Hom}(X, Y) \rightarrow \operatorname{Hom}([X],[Y])$ which sends a map $f$ to the class $[f]$ of the structure sheaf of its graph. It is compatible with composition and defines a functor $X \mapsto[X]$ from the category of separated schemes to the category of correspondences.

A homotopy of correspondences from $X$ to $Y$ will be an element of $\operatorname{Hom}\left(\left[X \times \mathbb{A}^{1}\right],[Y]\right)$. If two correspondences $f$ and $g$ are homotopic, we'll write $f \sim g$. Composition preserves homotopies.

We are particularly interested in correspondences $[X] \rightarrow\left[\mathbb{G}_{m}\right]$. We can use companion matrices to construct them. Let $f=T^{n}+a_{n-1} T^{n-1}+\cdots+a_{0} \in$ $R[T]$ be a polynomial with unit constant term $a_{0} \in R^{\times}$. Since $f$ is monic, as an $R$-module $P$ is free with rank equal to $n$. Since $T$ acts invertibly on $P:=R[T] / f$, we can regard $P$ as a finitely generated $R\left[T, T^{-1}\right]$-module, hence as an object of $\mathcal{P}\left(X, \mathbb{G}_{m}\right)$. The eigenvalues of $T$ acting on $P$ are the roots of $f$, so let's use the notation $[f=0]$ for the correspondence $[P]$.

Lemma 6.1. If $f$ and $g$ are monic polynomials with unit constant term, then $[f=0]+[g=0] \sim[f g=0]:[X] \rightarrow\left[\mathbb{G}_{m}\right]$.

Proof. The exact sequence

$$
0 \rightarrow R\left[T, T^{-1}\right] / f \rightarrow R\left[T, T^{-1}\right] / f g \rightarrow R\left[T, T^{-1}\right] / g \rightarrow 0
$$

splits up to homotopy, according to 5.1 .

If $a \in R^{\times}$is a unit, it may be regarded as a map $a: X \rightarrow \mathbb{G}_{m}$, and then $[a]=[T-a=0]$.

Lemma 6.2. If $a, b \in R^{\times}$, then $[a b] \sim[a]+[b]-[1]:[X] \rightarrow\left[\mathbb{G}_{m}\right]$.

Proof. (See $[41,4.6 .1]$.) We compute $[a]+[b]=[T-a=0]+[T-b=0] \sim$ $[(T-a)(T-b)=0]=\left[T^{2}-(a+b) T+a b=0\right] \sim\left[T^{2}-(1+a b) T+a b=\right.$ $0]=[(T-1)(T-a b)=0] \sim[(T-1)]+[(T-a b)=0]=[1]+[a b]$. The homotopy in the middle arises from adjoining a new variable $V$ and using the homotopy $\left[T^{2}-(V(a+b)+(1-V)(1+a b)) T+a b=0\right]$, which is valid because its constant term $a b$ is a unit. 
Now consider correspondences of the form $[X] \rightarrow\left[\mathbb{G}_{m} \times \mathbb{G}_{m}\right]$. The simplest ones are obtained from maps $X \rightarrow \mathbb{G}_{m} \times \mathbb{G}_{m}$, i.e., from pairs $(b, c)$ of units in $R$. Let $[b, c]$ denote such a correspondence.

Lemma 6.3. If $a, b, c \in R^{\times}$, then $[a b, c] \sim[a, c]+[b, c]-[1, c]:[X] \rightarrow$ $\left[\mathbb{G}_{m} \times \mathbb{G}_{m}\right]$.

Proof. (See $[41,4.6 .2]$.) The function $\operatorname{Hom}\left([X],\left[\mathbb{G}_{m}\right]\right) \rightarrow \operatorname{Hom}\left([X],\left[\mathbb{G}_{m} \times \mathbb{G}_{m}\right]\right)$ defined by $f \mapsto(f \otimes[c]) \circ[D]$, where $D$ is the diagonal embedding $X \rightarrow X \times X$, sends $[a]$ to $[a, c]$ and preserves homotopies.

Since $\operatorname{Hom}([X],[Y])$ is an abelian group, the convention introduced above for applying functors to $\mathbb{S}^{1}$ or to $\mathbb{S}^{t}$ applies, and we can attach a meaning to $\operatorname{Hom}\left([X],\left[\mathbb{S}^{1}\right]\right)$ or to $\operatorname{Hom}\left(\left[\mathbb{S}^{1}\right],[Y]\right)$. If we enlarge the category of correspondences slightly by taking its idempotent completion we may even interpret $\left[\mathbb{S}^{1}\right]$ as an object of the category of correspondences. To do so we introduce new objects denoted by $p[X]$ whenever $X$ is a scheme and $p \in \operatorname{Hom}([X],[X])$ is an idempotent, i.e., satisfies the equation $p^{2}=p$. We define $\operatorname{Hom}(p[X], q[Y]):=q \operatorname{Hom}([X],[Y]) p \subseteq \operatorname{Hom}([X],[Y])$, and with this definition composition is nothing new. We define $p[X] \otimes q[Y]:=(p \otimes q)[X \times Y]$. A homotopy between maps $p[X] \rightrightarrows q[Y]$ will be a map $p[X] \otimes\left[\mathbb{A}^{1}\right] \rightarrow q[Y]$.

A map $f:[X] \rightarrow[Y]$ may be said to induce the map $q f p: p[X] \rightarrow q[Y]$, but that procedure is not necessarily compatible with composition, for in terms of matrices it is the function $\left(\begin{array}{ll}a & b \\ c & d\end{array}\right) \mapsto a$. Nevertheless, we will abuse notation slightly and denote that induced map by $f: p[X] \rightarrow q[Y]$, leaving it to the reader to understand the necessity of composing $f$ with $p$ and $q$. Similarly, if we have an equation $f=g: p[X] \rightarrow q[Y]$ or a homotopy $f \sim g: p[X] \rightarrow q[Y]$, we'll understand that both $f$ and $g$ are to treated that way.

We identify $[X]$ with $1[X]$, and prove easily that $[X] \cong p[X] \oplus \bar{p}[X]$, where $\bar{p}:=1-p$. Any functor $F$ from the old category of correspondences to the category of abelian groups can be extended to the new category by defining $F(p[X]):=F(p) F([X])$. For example, with this notation, $F\left(\left[\mathbb{S}^{1}\right]\right)=F\left(\bar{e}\left[\mathbb{G}_{m}\right]\right)$, where $e$ is the composite map $\mathbb{G}_{m} \rightarrow$ Spec $\mathbb{Z} \stackrel{*}{\rightarrow} \mathbb{G}_{m}$. Thus we may as well identify $\left[\mathbb{S}^{1}\right]$ with $\bar{e}\left[\mathbb{G}_{m}\right]$, and similarly, $\left[\mathbb{S}^{2}\right]$ with $\left[\mathbb{S}^{1}\right] \otimes\left[\mathbb{S}^{1}\right]=(\bar{e} \otimes \bar{e})\left[\mathbb{G}_{m} \times \mathbb{G}_{m}\right]$.

Lemma 6.4. Suppose $a, b, c \in R^{\times}$. Then $[a b] \sim[a]+[b]:[X] \rightarrow\left[\mathbb{S}^{1}\right]$ and $[a b, c] \sim[a, c]+[b, c]:[X] \rightarrow\left[\mathbb{S}^{2}\right]$

Proof. Recall our convention about composing with idempotents when necessary. Use 6.2 to get $\bar{e}[a b] \sim \bar{e}([a]+[b]-[1])=\bar{e}([a]+[b])-\bar{e}[1]$ and then compute $\bar{e}[1]=[1]-[1]=0$. Use 6.3 to get $(\bar{e} \otimes \bar{e})[a b, c] \sim(\bar{e} \otimes \bar{e})([a, c]+[b, c]-[1, c])$ and then compute $(\bar{e} \otimes \bar{e})[1, c]=[1, c]-[1, c]-[1,1]+[1,1]=0$.

Lemma 6.5. Let $T, U$ be the standard coordinates on $\mathbb{G}_{m} \times \mathbb{G}_{m}$. Then $[T U] \sim$ $0:\left[\mathbb{S}^{2}\right] \rightarrow\left[\mathbb{G}_{m}\right]$. 
Proof. We compute $[T U](\bar{e} \otimes \bar{e})=[T U]-[T U](1 \otimes e)-[T U](e \otimes 1)+[T U](e \otimes$ $e)=[T U]-[T]-[U]+[1] \sim 0$, applying 6.2.

Corollary 6.6. $[T U,-T U] \sim 0:\left[\mathbb{S}^{2}\right] \rightarrow\left[\mathbb{G}_{m}^{2}\right]$.

Proof. Compose the result of the lemma with the map $\mathbb{G}_{m} \rightarrow \mathbb{G}_{m}^{2}$ defined by $v \mapsto(v,-v)$.

Corollary 6.7. $[T, U]+[U, T] \sim 0:\left[\mathbb{S}^{2}\right] \rightarrow\left[\mathbb{G}_{m}^{2}\right]$.

Proof. Compute $0 \sim[T U,-T U] \sim[T,-T U]+[U,-T U] \sim[T,-T]+[T, U]+$ $[U, T]+[U,-U]:\left[\mathbb{S}^{2}\right] \rightarrow\left[\mathbb{G}_{m}^{2}\right]$ and then observe that $[T,-T] \sim 0 \sim[U,-U]:$ $\left[\mathbb{S}^{2}\right] \rightarrow\left[\mathbb{G}_{m}^{2}\right]$, because, for example $[T,-T](\bar{e} \otimes \bar{e})=[T,-T]-[T,-T]-[1,-1]+$ $[1,-1]=0$.

Corollary 6.8. $[T, U] \sim\left[U^{-1}, T\right]:\left[\mathbb{S}^{2}\right] \rightarrow\left[\mathbb{G}_{m}^{2}\right]$.

Proof. Compute $0=[1, T]=\left[U U^{-1}, T\right] \sim[U, T]+\left[U^{-1}, T\right]:\left[\mathbb{S}^{2}\right] \rightarrow\left[\mathbb{G}_{m}^{2}\right]$, and then apply 6.7 .

Corollary 6.9. $\left[U^{-1}, T\right] \sim 1:\left[\mathbb{S}^{2}\right] \rightarrow\left[\mathbb{S}^{2}\right]$.

Proof. Use 6.8 and observe that $[T, U]:\left[\mathbb{S}^{2}\right] \rightarrow\left[\mathbb{S}^{2}\right]$ is the identity map.

The map $\left[U^{-1}, T\right]:\left[\mathbb{S}^{2}\right] \rightarrow\left[\mathbb{S}^{2}\right]$ is an analogue of the signed permutation $\left(\begin{array}{cc}0 & -1 \\ 1 & 0\end{array}\right)$ considered in Remark 5.2, and the homotopy found in Corollary 6.9 gives us a switching swindle that can be used to prove the cancellation theorem.

Having done that, the next task is to construct the map $\rho$ used in (6.2): it doesn't quite exist (!), but one can proceed as follows. Suppose $P \in \mathcal{P}(X \times$ $\left.\mathbb{S}^{1}, Y \times \mathbb{S}^{1}\right)$, so $P$ is an $R\left[T, T^{-1}\right]-S\left[V, V^{-1}\right]$-module that is finitely generated and projective as an $R\left[T, T^{-1}\right]$-module. We try to define $\rho[P]:=\left[P /\left(T^{n}-1\right)\right]-$ $\left[P /\left(T^{n}-V\right)\right] \in K_{0}^{\oplus}(\mathcal{P}(X, Y))$. It's easy to check that $P /\left(T^{n}-1\right)$ is a projective $R$-module. It turns out that for $n$ sufficiently large, multiplication by $T^{n}-V$ on $P$ is injective, and the bound is independent of the ring, so injectivity is preserved by base change, implying that $P /\left(T^{n}-V\right)$ is flat, hence projective as an $S$-module, since it's finitely generated. To show that $\rho$ is a left inverse for $\mu$ in (6.2), assume that $Q \in \mathcal{P}(X, Y)$ and let $P=Q \otimes_{R} R\left[T, T^{-1}\right]$ with $V$ acting on $P$ the same way $T$ does, and compute $\rho[P]=\left[Q^{n}\right]-\left[Q^{n-1}\right]=$ $[Q]$. One handles the problem of choosing $n$ in the definition of $\rho$ by explicit computations with cycle classes.

The usual definition of motivic cohomology is this: the chain complex $\mathbb{Z}^{\text {cor }}(t)$, usually called $\mathbb{Z}(t)$, is defined as $\mathbb{Z}^{\text {ex }}(t)$ was, but $K_{0}(\mathcal{P}(X, Y))$ is replaced by the group $\operatorname{Cor}(X, Y)$ of finite correspondences, those algebraic cycles on $X \times Y$ each component of which maps finitely and surjectively onto some irreducible component of $Y$. See [46] for the equivalence between $\mathbb{Z}^{\text {cor }}(t)$ and $\mathbb{Z}^{\mathrm{Bl}}(i)$. There is a natural map $\mathbb{Z}^{\mathrm{ex}}(t) \rightarrow \mathbb{Z}^{\mathrm{cor}}(t)$, which sends a coherent sheaf 
to its support cycle; according to [50, Corollary 6.32] the map is an equivalence. Thus in order for the spectral sequence (5.1) to be useful, the main remaining problem is to show that the map $\mathbb{Z}^{\oplus}(t) \rightarrow \mathbb{Z}^{\operatorname{ex}}(t)$ is an equivalence, or equivalently, that $\mathbb{Z}^{\oplus}(t) \rightarrow \mathbb{Z}^{\text {cor }}(t)$ is an equivalence.

Now let's sketch the rest of the proof of Suslin's theorem in [41] that, locally, for smooth varieties over a field, that the map $\mathbb{Z}^{\oplus}(t) \rightarrow \mathbb{Z}^{\text {cor }}(t)$ is an equivalence.

The basic strategy is to prove that the groups $H^{*}\left(X, \mathbb{Z}^{\oplus}(t)\right)$ share enough properties with motivic cohomology $H^{*}\left(X, \mathbb{Z}^{\text {cor }}(t)\right)$ that they must be equal. First comes the statement that $H^{k}\left(X, \mathbb{Z}^{\oplus}(t)\right) \cong H^{k+1}\left(X \times \mathbb{S}^{1}, \mathbb{Z}^{\oplus}(t+1)\right)$, which follows from cancellation. (It corresponds to the part of the Fundamental Theorem of algebraic $K$-theory that says that $K_{n}(R) \cong K_{n+1}\left(\operatorname{Spec} R \times \mathbb{S}^{1}\right)$ for a regular noetherian ring $R$.) Relating $\mathbb{S}^{1}$ to $\mathbb{G}_{m}$, which is an open subset of $\mathbb{A}^{1}$, yields a statement about cohomology with supports, $H^{k}\left(X, \mathbb{Z}^{\oplus}(t)\right) \cong$ $H_{X \times 0}^{k+2}\left(X \times \mathbb{A}^{1}, \mathbb{Z}^{\oplus}(t+1)\right)$, for $k \geq 0$. By induction one gets $H^{k}\left(X, \mathbb{Z}^{\oplus}(t)\right) \cong$ $H_{X \times 0}^{k+2 m}\left(X \times \mathbb{A}^{m}, \mathbb{Z}^{\oplus}(t+m)\right)$ for $m \geq 0$. This works also for any vector bundle $E$ of rank $m$ over $X$, yielding $H^{k}\left(X, \mathbb{Z}^{\oplus}(t)\right) \cong H_{s(X)}^{k+2 m}\left(E, \mathbb{Z}^{\oplus}(t+m)\right)$, where $s: X \rightarrow E$ is the zero section. Now take a smooth closed subscheme $Z$ of $X$ of codimension $m$, and use deformation to the normal bundle to get $H^{k}\left(Z, \mathbb{Z}^{\oplus}(t)\right) \cong H_{Z}^{k+2 m}\left(X, \mathbb{Z}^{\oplus}(t+m)\right)$.

Now it's time to show that the map $H_{Z}^{*}\left(X, \mathbb{Z}^{\oplus}(t)\right) \rightarrow H_{Z}^{*}\left(X, \mathbb{Z}^{\text {cor }}(t)\right)$ is an isomorphism, but it's still not easy. The multi-relative cohomology groups with supports, $H_{Z}^{*}\left(\mathbb{A}^{m}, \partial \mathbb{A}^{m} ; \mathbb{Z}^{\oplus}(t)\right)$ arise (analogous to the multi-relative $K$ groups described in section 7 ), and Suslin develops a notion he calls "rationally contractible presheaves" to handle the rest of the proof.

\section{Higher Chow groups and a motivic spectral sequence}

In this section we describe Bloch's approach to motivic cohomology via higher Chow groups and then describe the argument of Bloch and Lichtenbaum for a motivic spectral sequence.

The definition of linear equivalence of algebraic cycles amounts to saying that there is the following exact sequence, in which the two arrows are derived from the inclusion maps $\mathbb{A}^{0} \rightrightarrows \mathbb{A}^{1}$ corresponding to the points 0 and 1 .

$$
C H^{i}(X) \leftarrow \mathcal{Z}^{i}(X) \leftleftarrows \mathcal{Z}^{i}\left(X \times \mathbb{A}^{1}\right)
$$

The first step in developing motivic cohomology groups to serve in (1.2) or (3.2) is to bring homological algebra to bear: evidently $C H^{i}(X)$ is a cokernel, and in homological algebra one can't consider a cokernel without also considering the kernel. One way to handle that is to try to continue the sequence above forever, forming a chain complex like the one in (7.2).

$$
\mathcal{Z}^{i}(X) \leftarrow \mathcal{Z}^{i}\left(X \times \mathbb{A}^{1}\right) \leftarrow \mathcal{Z}^{i}\left(X \times \mathbb{A}^{2}\right) \leftarrow \cdots
$$


For this purpose, Bloch used the cosimplicial affine space $\mathbb{A}^{*}$, introduced earlier, and algebraic cycles on the corresponding cosimplicial variety $X \times \mathbb{A}$. He defined $\mathcal{Z}^{i}(X, n) \subseteq \mathcal{Z}^{i}\left(X \times \mathbb{A}^{n}\right)$ to be the group of algebraic cycles of codimension $i$ on $X \times \mathbb{A}^{n}$ meeting all the faces properly. (Two subvarieties are said to meet properly if the codimension of the intersection is the sum of the codimensions of the subvarieties.) The result is a simplicial abelian group $n \mapsto \mathcal{Z}^{i}(X, n)$. Its face maps involve intersecting a cycle with a hyperplane, keeping track of intersection multiplicities. Bloch defined the higher Chow group $C H^{i}(X, n)$ as its homology group in degree $n$, and we'll introduce the auxiliary notation $H^{2 i-n}\left(X, \mathbb{Z}^{\mathrm{Bl}}(i)\right)$ for it here to make the anticipated use as motivic cohomolgy more apparent. A useful abuse of notation is to write $\mathbb{Z}^{\mathrm{Bl}}(i)=\left(n \mapsto \mathcal{Z}^{i}(X, 2 i-n)\right)$, leaving it to the reader to remember to replace the simplicial abelian group by its associated chain complex. We let $H^{2 i-n}\left(X, \mathbb{Q}^{B l}(i)\right):=H^{2 i-n}\left(X, \mathbb{Z}^{\mathrm{Bl}}(i)\right) \otimes \mathbb{Q}$.

A good survey of results about Bloch's higher Chow groups is available in [27, II, §2.1]; for the original papers see [6], [7], and [26]. The main results are that $H^{n}\left(X, \mathbb{Z}^{\mathrm{Bl}}(i)\right)$ is homotopy invariant, fits into a localization sequence for an open subscheme and its complement, can be made into a contravariant functor (on nonsingular varieties), and can be compared rationally with $K$ theory.

For example, in [7] Bloch proves a moving lemma which implies a localization theorem for $\mathbb{Z}^{\mathrm{Bl}}$. Suppose $X$ is quasiprojective, let $U \subseteq X$ be an open subset, let $Z=X-U$, and assume $Z$ has codimension $p$ in $X$. The localization theorem provides the following long exact sequence.

$$
\begin{aligned}
\cdots & \rightarrow H^{j-1}\left(U, \mathbb{Z}^{\mathrm{Bl}}(i)\right) \rightarrow H^{j-2 p}\left(Z, \mathbb{Z}^{\mathrm{Bl}}(i-p)\right) \rightarrow H^{j}\left(X, \mathbb{Z}^{\mathrm{Bl}}(i)\right) \\
& \rightarrow H^{j}\left(U, \mathbb{Z}^{\mathrm{Bl}}(i)\right) \rightarrow \cdots
\end{aligned}
$$

In $[6,9.1]$ Bloch presents a comparison isomorphism

$$
\tau: K_{n}^{\prime}(X)_{\mathbb{Q}} \cong \bigoplus_{d} H^{2 i-n}\left(X, \mathbb{Q}^{B l}(i)\right)
$$

for $X$ a quasiprojective variety over a field $k$. The proof (with some flaws corrected later) proceeds by using the localization theorem to reduce to the case where $X$ is affine and smooth over $k$, then uses relative $K$-theory to reduce to the case where $m=0$, and finally appeals to Grothendieck's result (1.1). In the case where $X$ is nonsingular the isomorphism $\tau$ differs from the higher Chern character map ch by multiplication by the Todd class of $X$, which is a unit in the Chow ring, so Bloch's result implies that the map ch : $K_{n}(X)_{\mathbb{Q}} \stackrel{\cong}{\longrightarrow} \bigoplus_{d} H^{2 i-n}\left(X, \mathbb{Q}^{B l}(i)\right)$ is an isomorphism, too. In $[26,3.1]$ Levine proves the same result, but avoids Chern classes by using a more detailed computation of the relative $K$-groups. Finally, a detailed summary of the complete proof when $X$ is nonsingular is available in [27, III, §3.6].

Now we sketch some details of the argument from the unpublished preprint [8] for a motivic spectral sequence for the spectrum of a field $k$. 
A cube of dimension $n$ is a diagram (in some category) indexed by the partially ordered set of subsets of the set $\{1,2, \ldots, n\}$. The homotopy fiber hofib $C$ of a cube $C$ of dimension $n$ of spaces or spectra can be constructed inductively as follows. When $n=0$, hofib $C$ is the single space in the diagram. For $n>0$ consider the two cubes $C^{\prime}$ and $C^{\prime \prime}$ of dimension $n-1$ appearing as faces perpendicular to a chosen direction and take hofib $C$ to be the homotopy fiber of the map hofib $C^{\prime} \rightarrow$ hofib $C^{\prime \prime}$.

If $Y_{1}, \ldots, Y_{n}$ are open or closed subschemes of a scheme $X$, their intersections $Y_{i_{1}} \cap \cdots \cap Y_{i_{k}}$ form a cube of schemes. Applying the $K$-theory functor (which is contravariant) gives a cube of spectra. When $Y_{1}, \ldots, Y_{n}$ are closed subschemes, the homotopy fiber of the cube is denoted by $K\left(X ; Y_{1}, \ldots, Y_{n}\right)$ and is called multi-relative $K$-theory. If $Y_{1}=X-V$ is an open subscheme of $X$, the homotopy fiber of the cube is denoted by $K^{V}\left(X ; Y_{2}, \ldots, Y_{n}\right)$, and is called multi-relative $K$-theory with supports in $V$. If $\mathcal{V}$ is a family of closed subsets of $X$ that is closed under finite unions, then the induc-

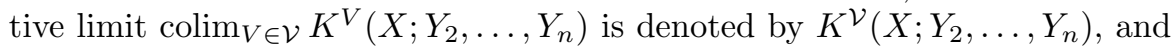
is called multi-relative $K$-theory with supports in $\mathcal{V}$. If $\mathcal{W}$ is another such family of closed subsets of $X$, then $\operatorname{colim}_{W \in \mathcal{W}} \operatorname{colim}_{V \in \mathcal{V}} K^{V-W}\left(X-W ; Y_{2}-\right.$ $\left.W, \ldots, Y_{n}-W\right)$ is denoted by $K^{\mathcal{V}-\mathcal{W}}\left(X ; Y_{2}, \ldots, Y_{n}\right)$, which one might call multi-relative $K$-theory with supports in $\mathcal{V}$ away from $\mathcal{W}$. Finally, if $p \in \mathbb{Z}$, we let $K_{p}^{\mathcal{V}-\mathcal{W}}\left(X ; Y_{2}, \ldots, Y_{n}\right)$ be the homotopy group $\pi_{p} K^{\mathcal{V}-\mathcal{W}}\left(X ; Y_{2}, \ldots, Y_{n}\right)$, and similarly for the other notations.

The notation for multi-relative $K$-theory is applied to the simplices $\mathbb{A}^{p}=$ $\mathbb{A}_{k}^{p}$ as follows. For a variety $X$ and for any $p \geq 0$ we let $\mathcal{V}^{n}$ denote the family of closed subsets of $X \times \mathbb{A}^{p}$ which are finite unions of subvarieties of codimension $n$ meeting $X \times \mathbb{A}^{m}$ properly, for each face $\mathbb{A}^{m} \subseteq \mathbb{A}^{p}$ of the simplex $\mathbb{A}^{p}$. In this section we are interested in the case where $X=\operatorname{Spec} k$.

When $K$-theory (or multirelative $K$-theory) with supports in $\mathcal{V}^{n}$ is intended, we'll write $K^{(n)}$ in place of $K^{\mathcal{\nu}^{n}}$. The spectrum $K^{(n)}\left(X \times \mathbb{A}^{p}\right)$ is functorial in the sense that if $\mathbb{A}^{q} \rightarrow \mathbb{A}^{p}$ is an affine map sending vertices to vertices, there is an induced map $K^{(n)}\left(X \times \mathbb{A}^{p}\right) \rightarrow K^{(n)}\left(X \times \mathbb{A}^{q}\right)$. These maps can be assembled to form a simplicial space $p \mapsto K^{(n)}\left(X \times \mathbb{A}^{p}\right)$ which we'll call $K^{(n)}\left(X \times \mathbb{A}^{*}\right)$.

Let $H_{0}, \ldots, H_{p}$ be the codimension 1 faces of $\mathbb{A}^{p}$. Let $\partial \mathbb{A}^{p}$ be an abbreviation for the sequence $H_{0}, \ldots, H_{p}$, and let $\Sigma$ be an abbreviation for the sequence $H_{0}, \ldots, H_{p-1}$.

The main result is [8, Theorem 1.3.3], which states that the following sequence of multi-relative $K_{0}$ groups is exact.

$$
\begin{aligned}
\ldots & \rightarrow K_{0}^{(n+1)}\left(\mathbb{A}^{p} ; \partial \mathbb{A}^{p}\right) \stackrel{i}{\rightarrow} K_{0}^{(n)}\left(\mathbb{A}^{p} ; \partial \mathbb{A}^{p}\right) \rightarrow K_{0}^{(n)}\left(\mathbb{A}^{p} ; \Sigma\right) \\
& \rightarrow K_{0}^{(n)}\left(\mathbb{A}^{p-1} ; \partial \mathbb{A}^{p-1}\right) \rightarrow K_{0}^{(n-1)}\left(\mathbb{A}^{p-1} ; \partial \mathbb{A}^{p-1}\right) \rightarrow \ldots
\end{aligned}
$$

The proof of exactness hinges on showing that the related map

$$
K_{0}^{\mathcal{V}^{n}-\mathcal{V}^{n+1}}\left(\mathbb{A}^{p} ; \partial \mathbb{A}^{p}\right) \rightarrow K_{0}^{\mathcal{V}^{n}-\mathcal{V}^{n+1}}\left(\mathbb{A}^{p} ; \Sigma\right)
$$


is injective, which in turn depends on a moving lemma that occupies the bulk of the paper. Two of every three terms in the long exact sequence look like $K_{0}^{(n)}\left(\mathbb{A}^{p} ; \partial \mathbb{A}^{p}\right)$ with a change of index, so it's actually an exact couple. The abutment of the corresponding spectral sequence is the colimit of the chain of maps like the one labelled $i$ above, and thus is $K_{0}\left(\mathbb{A}^{p} ; \partial \mathbb{A}^{p}\right)$, which, by an easy computation, turns out to be isomorphic to $K_{p}(k)$. The $E_{1}$ term $K_{0}^{\mathcal{V}^{n}}\left(\mathbb{A}^{p} ; \Sigma\right)$ is isomorphic to $K_{0}^{\mathcal{V}^{n}-\mathcal{V}^{n+1}}\left(\mathbb{A}^{p} ; \Sigma\right)$, which in turn is isomorphic to the subgroup of $\mathcal{Z}^{n}(X, p)$ consisting of cycles which pull back to 0 in each face mentioned in $\Sigma$; it follows that the $E_{2}$ term is $C H^{n}(X, p)$. The final result of Bloch and Lichtenbaum is the following theorem.

Theorem 7.1. If $k$ is a field and $X=$ Spec $k$, then there is a motivic spectral sequence of the following form.

$$
E_{2}^{p q}=H^{p-q}\left(X, \mathbb{Z}^{B l}(-q)\right) \Rightarrow K_{-p-q}(X)
$$

We also mention $[16, \S 7]$, which shows that the filtration of the abutment provided by this spectral sequence is the $\gamma$-filtration and that the spectral sequence degenerates rationally.

We continue this line of development in section 8 .

\section{Extension to the global case}

In this section we sketch the ideas of Friedlander and Suslin [12] for generalizing the Bloch-Lichtenbaum spectral sequence, Theorem 7.1, to the global case, in other words, to establish it for any nonsingular variety $X$. The first step is to show that the Bloch-Lichtenbaum spectral sequence arises from a filtration of the $K$-theory spectrum, and that the successive quotients are Eilenberg-MacLane spectra. For the subsequent steps, Levine gives an alternate approach in [28], which we also sketch briefly.

The paper [12] is a long one, so it will be hard to summarize it here, but it is carefully written, with many foundational matters spelled out in detail.

Let $\Delta$ be the category of finite nonempty ordered sets of the form $[p]:=$ $\{0,1,2, \ldots, p\}$ for some $p$, so that a simplicial object is a contravariant functor from $\Delta$ to some other category. Since any finite nonempty ordered set is isomorphic in a unique way to some object of $\Delta$, we can think of a simplicial object as a functor on the category of all finite nonempty ordered sets.

Recall that the relative $K$-group $K_{0}^{(n)}\left(\mathbb{A}^{p} ; \partial \mathbb{A}^{p}\right)$ is constructed from a cube whose vertices are indexed by the intersections of the faces of the simplex $\mathbb{A}^{p}$. Any such intersection is a face itself, unless it's empty. The faces are indexed by the nonempty subsets of the ordered set $[p]$, so the intersections of faces are indexed by the subsets of $[p]$ (including the empty subset), and those subsets correspond naturally to the vertices of a $p+1$-dimensional cube. The $K$-theory space of the empty scheme is the $K$-theory space of the zero 
exact category, so is a one point space. If $Z$ is a pointed simplicial space (or spectrum), then by defining $Z(\phi)$ to be the one point space, we can apply it to the cube of subsets of $[p]$, yielding a $p+1$-dimensional cube of spaces (or spectra) we'll call cube ${ }_{p+1} Z$. As in [12, A.1] we see that this procedure, when applied to $K^{(n)}\left(\mathbb{A}^{*}\right)=\left(q \mapsto K^{(n)}\left(\mathbb{A}^{q}\right)\right)$, yields a cube whose homotopy fiber is $K^{(n)}\left(\mathbb{A}^{p} ; \partial \mathbb{A}^{p}\right)$.

It is well known that homotopy cofibers (mapping cones) and homotopy fibers amount to the same thing (with a degree shift of 1) for spectra. Given an $m$-dimensional cube $Y$ of pointed spaces form the homotopy cofiber hocofib $Y$ : the construction is inductive, as for the homotopy fiber of a cube described above, but at each stage the mapping cone replaces the homotopy fiber of a map. Friedlander and Suslin relate the homotopy fiber to the homotopy cofiber by proving that the natural map hofib $Y \rightarrow \Omega^{m}$ hocofib $Y$ is a $2 m-N+1$ equivalence if each space in the cube $Y$ is $N$-connected $[12,3.4]$. The proof uses the Freudenthal Suspension theorem and the Blakers-Massey Excision theorem.

A cube of the form cube ${ }_{m} Z$ arising from a simplicial space $Z$ comes with an interesting natural map

$$
\text { hocofib cube }_{m} Z \rightarrow \Sigma|Z|
$$

(see [12, 2.6]), which for $N \geq 1$ is $N+m+1$-connected if each space $Z_{i}$ is $N$-connected $[12,2.11]$. The proof goes by using homology to reduce to the case where $Z$ is a simplicial abelian group.

Combining the two remarks above and passing to spectra, we get a map $K_{i}^{(n)}\left(\mathbb{A}^{p} ; \partial \mathbb{A}^{p}\right) \rightarrow \pi_{i+p} K^{(n)}\left(\mathbb{A}^{*}\right)$ which is an isomorphism for $i \leq-1$. It turns out that $i=0$ (the value occuring in the construction of Bloch and Lichtenbaum) is close enough to $i=-1$ so that further diagram chasing [12, §5-6] allows the exact couple derived from the exact couple of Bloch and Lichtenbaum to be identified with the exact couple arising from the filtration of spectra defined by $F^{n}:=\left|K^{(n)}\left(\mathbb{A}^{*}\right)\right|$, and for the successive quotients in the filtration to be identified with the Eilenberg-MacLane spectrum arising from the chain complex defining $\mathbb{Z}^{\mathrm{Bl}}$.

So much for the first step, which was crucial, since it brings topology into the game. Next the authors show that $K^{(n)}\left(X \times \mathbb{A}^{*}\right)$ is homotopy invariant in the sense that it doesn't change if $X$ is replaced by $X \times \mathbb{A}^{1}$; by induction, the same is true for the product with $\mathbb{A}^{m}$. Combining that with a result of Landsburg [25] establishes the motivic spectral sequence for $X=\mathbb{A}^{m}$. To go further, we take $X=\mathbb{A}^{m}$ and $m=n$ and examine $K^{(n)}\left(\mathbb{A}^{n} \times \mathbb{A}^{*}\right)$. Cycles on $\mathbb{A}^{n} \times \mathbb{A}^{m}$ that are quasifinite over $\mathbb{A}^{m}$ have codimension $n$. A moving lemma of Suslin asserts that such cycles are general enough, allowing us to replace $K^{(n)}\left(\mathbb{A}^{n} \times \mathbb{A}^{*}\right)$ by $K^{\mathcal{Q}}\left(\mathbb{A}^{n} \times \mathbb{A}^{*}\right)$, where $\mathcal{Q}$ denotes the family of support varieties that are quasifinite over the base. The advantage here is increased functoriality, since quasifiniteness is preserved by base change. Transfer maps can also be defined, allowing the globalization theorem of Voevodsky for "pretheories" 
to be adapted for the current situation, thereby establishing the spectral sequence when $X$ is a smooth affine semilocal variety over a field. Finally, the globalization techniques of Brown and Gersten [9] involving hypercohomology of sheaves of spaces are used to establish the spectral sequence when $X$ is a smooth affine variety.

Levine's approach [28] to globalizing the spectral sequence is somewhat different. Instead of developing transfer maps as Friedlander and Suslin do, he replaces the $K$-theory of locally free sheaves by the $K$-theory of coherent sheaves (which is called $G$-theory) so singular varieties can be handled. Then he develops a localization theorem [28, Corollary 7.10] that provides, when $U$ is an open subvariety of a variety $X$, a fibration sequence $G^{(n)}\left((X-U) \times \mathbb{A}^{\cdot}\right) \rightarrow$ $G^{(n)}\left(X \times \mathbb{A}^{\cdot}\right) \rightarrow G^{(n)}\left(U \times \mathbb{A}^{*}\right)$. The proof of the localization theorem involves a very general moving lemma [28, Theorem 0.9$]$ that, roughly speaking, takes a cycle on $U \times \mathbb{A}^{N}$ meeting the faces properly and moves it to a cycle whose closure in $X$ still meets the faces properly. But what's really "moving" is the ambient affine space, which is blown up repeatedly along faces - the blow-ups are then "trianglated" by more affine spaces.

Levine's final result is more general than the Friedlander-Suslin version, since it provides a motivic spectral sequence for any smooth scheme $X$ over a regular noetherian scheme of dimension 1 .

\section{The slice filtration}

In this section we sketch some of Voevodsky's ideas [43, 47, 49] aimed at producing a motivic spectral sequence. The setting is Voevodsky's $\mathbb{A}^{1}$-homotopy theory for schemes [32], which can be briefly described as follows. Modern algebraic topology is set in the world of simplicial sets or their geometric realizations; the spaces to be studied can be viewed as being obtained from colimits of diagrams of standard simplices $\Delta^{n}$, where the arrows in the diagram are affine maps that send vertices to vertices and preserve the ordering of the vertices. Voevodsky enlarges the notion of "space" by considering a field $k$ (or more generally, a noetherian finite dimensional base scheme), replacing the simplices $\Delta^{n}$ by affine spaces $\mathbb{A}_{k}^{n}$ over $k$, and throwing in all smooth varieties over $k$, as well as all colimits of diagrams involving such varieties. The colimits may be realized in a universal way as presheaves on the category of smooth varieties over $k$. These presheaves are then sheafified in the Nisnevich topology, a topology intermediate between the Zariski and étale topologies. Finally, the affine "simplices" $\mathbb{A}_{k}^{n}$ are forced to be contractible spaces. The result is the $\mathbb{A}^{1}$-homotopy category, a world where analogues of the techniques of algebraic topology have been developed. Spectra in this world are called motivic spectra. In this world there are two types of circles, the usual topological circle $\mathbf{S}_{s}^{1}$ and the algebraic circle $\mathbf{S}_{t}^{1}:=\operatorname{Spec}\left(k\left[U, U^{-1}\right]\right)$, so the spheres $\mathbf{S}^{n, i}:=\mathbf{S}_{s}^{n-i} \wedge \mathbf{S}_{t}^{i}$ have an extra index, as do the motivic spectra and the generalized cohomology theories they represent. The sphere $T:=\mathbf{S}^{2,1}$, half 
topological and half algebraic, turns out to be equivalent to the projective line $\mathbb{P}^{1}$, so the Fundamental Theorem of algebraic $K$-theory, which identifies $K\left(\mathbb{P}_{A}^{1}\right)$ with $K(A) \times K(A)$ for a ring $A$, shows that the motivic spectrum $K G L$ representing $K$-theory is $(2,1)$-periodic.

Voevodsky's idea for constructing a motivic spectral sequence is to build a filtration of the motivic spectrum $K G L$, in which each term $F^{p} K G L$ is to be a motivic spectrum. As in Goodwillie's remark in section 4, the filtration is to be compatible with products, the cofiber $F^{0 / 1} K G L$ is to be equivalent to the motivic analogue $H_{\mathbb{Z}}$ of the Eilenberg-MacLane ring spectrum associated to

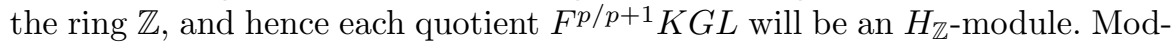
ulo problems with convergence, filtrations of motivic spectra yield spectral sequences as before.

The slice filtration of any motivic spectrum $Y$ is introduced in $[43, \S 2]$. The spectrum $F^{q} Y$ is obtained from $Y$ as that part of it that can be constructed from $(2 q, q)$-fold suspensions of suspension spectra of smooth varieties. Smashing two motivic spheres amounts to adding the indices, so the filtration is compatible with any multiplication on $Y$. The layer $s_{p}(Y):=F^{p / p+1} Y$ is called the $p$-th slice of $Y$.

Voevodsky states a number of interlocking conjectures about slice filtrations of various standard spectra [43]. For example, Conjecture 1 states that the slice filtration of $H_{\mathbb{Z}}$ is trivial, i.e., the slice $s_{0}\left(H_{\mathbb{Z}}\right)$ includes the whole thing.

Conjecture 10 (the main conjecture) says $s_{0}(\mathbf{1})=H_{\mathbb{Z}}$, where $\mathbf{1}$ denotes the sphere spectrum. By compatibility with multiplication, a corollary would be that the slices of any motivic spectrum are modules over $H_{\mathbb{Z}}$.

Conjecture 7 says that $s_{0}(K G L)=H_{\mathbb{Z}}$. Since $K G L$ is $(2,1)$-periodic, Conjecture 7 implies that $s_{q}(K G L)=\Sigma^{2 q, q} H_{\mathbb{Z}}$, thereby identifying the $E_{2}$ term of the spectral sequence, and providing a motivic spectral sequence of the desired form. In [47] Voevodsky shows Conjecture 7 is implied by Conjecture 10 and a seemingly simpler conjecture that doesn't refer to $K$-theory or the spectrum representing it. In [49] Voevodsky proves Conjecture 10 over fields of characteristic 0, providing good evidence for the conjecture in general.

\section{Filtrations for general cohomology theories}

We'll sketch briefly some of Levine's ideas from [29] that lead to a new replacement for the spectral sequence construction of Bloch-Lichtenbaum [8].

Levine's homotopy coniveau filtration is defined for a contravariant functor $E$ from the category of smooth schemes over a noetherian separated scheme $S$ of finite dimension to the category of spectra, but for simplicity, since some of his results require it, we'll assume $S$ is the spectrum of an infinite field $k$.

In section 7 we defined $K$-theory with supports. The same definitions can be applied to the functor $E$ as follows. If $V$ is a closed subset of $X$, we let $E^{V}(X)$ denote the homotopy fiber of the map $E(X) \rightarrow E(X-V)$. If $\mathcal{V}$ is a 
family of closed subsets of $X$ that is closed under finite unions, then we let $E^{\mathcal{V}}(X)$ denote the inductive $\operatorname{limit}_{\operatorname{colim}} \in \mathcal{V} E^{V}(X)$.

For each smooth variety $X$ Levine provides a natural filtration $E(X)=$ $F^{0} E(X) \leftarrow F^{1} E(X) \leftarrow F^{2} E(X) \leftarrow \ldots$ of $E(X)$ as follows.

In section 7 we introduced the family of supports $\mathcal{V}^{n}$ on $X \times \mathbb{A}^{p}$. Levine modifies the definition of it slightly, considering it instead to be the family of closed subsets of $X \times \mathbb{A}^{p}$ of codimension at least $n$ meeting each face in a subset of codimension at least $n$ in that face; when $X$ is a quasiprojective variety it amounts to the same thing. We define $F^{n} E(X)=\left|p \mapsto E^{\mathcal{V}^{n}}\left(X \times \mathbb{A}^{p}\right)\right|$.

We say that $E$ is homotopy invariant if for all smooth varieties $X$, the map $E(X) \rightarrow E\left(X \times \mathbb{A}^{1}\right)$ is a weak homotopy equivalence. Homotopy invariance of $E$ ensures that the natural map $E(X) \rightarrow F^{0} E(X)$ is an equivalence, so the filtration above can be regarded as a filtration of $E(X)$. Taking homotopy groups and exact couples leads to a spectral sequence, as before, but the homotopy groups of $E$ should be bounded below if the spectral sequence is to converge. Levine's axiom 1 is homotopy invariance of $E$.

The terms $F^{n} E$ and the layers $F^{n / n+1} E$ of the filtration are contravariant functors from the category of smooth varieties (but with just the equidimensional maps) to the category of spectra, so the procedure can be iterated. In particular, we can consider $\left(F^{n} E\right)^{V}(X),\left(F^{n} E\right)^{\mathcal{V}}(X)$, and $F^{m} F^{n} E(X)$.

We say that $E$ satisfies Nisnevich excision if for any étale map $f: X^{\prime} \rightarrow$ $X$ and for any closed subset $V \subseteq X$ for which the map $f$ restricts to an isomorphism from $V^{\prime}:=f^{-1}(V)$ to $V$, it follows that the map $E^{V}(X) \rightarrow$ $E^{V^{\prime}}\left(X^{\prime}\right)$ is a weak homotopy equivalence. (A special case is where $f$ is the inclusion of an open subset $X^{\prime}$ of $X$ into $X$, so that $V^{\prime}=V \subseteq X^{\prime} \subseteq X$.) Levine's axiom 2 is Nisnevich excision for $E$.

The first main consequence of assuming that $E$ satisfies Nisnevich excision is the localization theorem $[29,2.2 .1]$, which, for a closed subset $Z$ of $X$, identifies $\left(F^{n} E\right)^{Z}(X)$ with $\left|p \mapsto E^{\mathcal{W}}\left(X \times \mathbb{A}^{p}\right)\right|$, where $\mathcal{W}$ is the family of closed subsets of $X \times \mathbb{A}^{p}$ that meet each face in a subset of codimension at least $n$ and are contained in $Z \times \mathbb{A}^{p}$. There is an analogous statement for the layers.

Define the $p$-fold $T$-loop space $\Omega_{T}^{p} E$ of $E$ by the formula $\left(\Omega_{T} E\right)(X):=$ $E^{X \times 0}\left(X \times \mathbb{A}^{p}\right)$. Levine's axiom 3 is that there is a functor $E^{\prime}$ satisfying axioms 1 and 2 and there is a natural weak equivalence $E \stackrel{\sim}{\longrightarrow} \Omega_{T}^{2} E^{\prime}$.

The first main consequence of axiom 3 (which implies axioms 1 and 2) is the moving lemma, which is phrased as follows. Let $f: Y \rightarrow X$ be a map of smooth varieties over $k$. Let $\mathcal{U}^{n}$ be the family of closed subsets $V \subseteq$ $X \times \mathbb{A}^{p}$ of codimension at least $n$ meeting the faces in sets of codimension at least $n$, whose pullbacks $f^{-1}(V)$ have the same property, and let $F_{f}^{n} E(X)=$ $\left|p \mapsto E^{\mathcal{U}^{n}}\left(X \times \mathbb{A}^{p}\right)\right|$. Levine's moving lemma states that, provided $X$ is a smooth variety of dimension $d$ admitting a closed embedding into $\mathbb{A}^{d+2}$ with 
trivial normal bundle ${ }^{3}$, the map $F_{f}^{n} E(X) \rightarrow F^{n} E(X)$ is a weak homotopy equivalence.

Finally, we say that $E$ is well-connected if it satisfies axioms 1,2 , and 3 , and: (1) for every smooth variety $X$ and every closed subset $W \subseteq X$, the spectrum $E^{W}(X)$ is -1-connected; and $(2) \pi_{n}\left(F^{0 / 1}\left(\Omega_{T}^{d} E\right)(F)\right)=0$ for every finitely generated field extension $F$ of $k$, for every $d \geq 0$, and for every $n \neq 0$.

The main virtue of a well-connected functor $E$ is that it allows computations in terms of cycles. Indeed, Levine defines a generalization of Bloch's higher Chow groups that is based on $E$, which enters into computations of the layers in the filtration.

The objects $E(X)$ are already spectra, but the additional $T$-loop space functor $\Omega_{T}$ (or a related version called $\Omega_{\mathbb{P}^{1}}$ ) allows for the possibility of considering spectra formed with respect to it, whose terms are spectra in the usual sense. The associated machinery allows Levine to compare his coniveau filtration with the slice filtration of Voevodsky in section 9 and conclude they are equal. Finally, he is able to construct a homotopy coniveau spectral sequence, analogous to the Atiyah-Hirzebruch spectral sequence, converging to the homotopy groups of $E(X)$ (suitably completed to ensure convergence). In the case where $E=K$ is $K$-theory itself, he checks that $K$ is well-connected and that the spectral sequence agrees with the Bloch-Lichtenbaum spectral sequence of section 7 as globalized by Friedlander-Suslin in section 8 .

\section{References}

1. D. Anderson, M. Karoubi, and J. Wagoner, Relations between higher algebraic K-theories, Algebraic K-theory, I: Higher K-theories (Proc. Conf., Battelle Memorial Inst., Seattle, Wash., 1972), Springer, Berlin, 1973, pp. 73-81. Lecture Notes in Math. Vol. 341.

2. M. F. Atiyah and F. Hirzebruch, Vector bundles and homogeneous spaces, Proc. Sympos. Pure Math., Vol. III, American Mathematical Society, Providence, R.I., 1961, pp. 7-38.

3. Michael Atiyah, K-Theory Past and Present, arXiv:math.KT/0012213.

4. Alexander Beilinson, Letter to Christophe Soulé, January 11, 1982, K-theory Preprint Archives, http://www.math.uiuc.edu/K-theory/0694/.

5. lems in mathematics, Vol. 24, Itogi Nauki i Tekhniki, Akad. Nauk SSSR Vsesoyuz. Inst. Nauchn. i Tekhn. Inform., Moscow, 1984, pp. 181-238.

6. Spencer Bloch, Algebraic cycles and higher K-theory, Adv. in Math. 61 (1986), no. 3, 267-304.

7. _ The moving lemma for higher Chow groups, J. Algebraic Geom. 3 (1994), no. 3, 537-568.

8. Spencer Bloch and Steve Lichtenbaum, A Spectral Sequence for Motivic Cohomology, http://www.math.uiuc.edu/K-theory/0062/, March 3, 1995.

${ }^{3}$ all small enough open subsets of $X$ have such an embedding 
9. Kenneth S. Brown and Stephen M. Gersten, Algebraic K-theory as generalized sheaf cohomology, Algebraic K-theory, I: Higher K-theories (Proc. Conf., Battelle Memorial Inst., Seattle, Wash., 1972), Springer, Berlin, 1973, pp. 266-292. Lecture Notes in Math., Vol. 341.

10. A. D. Elmendorf, I. Kriz, M. A. Mandell, and J. P. May, Rings, modules, and algebras in stable homotopy theory, Mathematical Surveys and Monographs, vol. 47, American Mathematical Society, Providence, RI, 1997, With an appendix by M. Cole.

11. Eric M. Friedlander, Christian Haesemeyer, and Mark E. Walker, Techniques, computations, and conjectures for semi-topological $K$-theory, http://www.math.uiuc.edu/K-theory/0621/, February 20, 2003.

12. Eric M. Friedlander and Andrei Suslin, The spectral sequence relating algebraic K-theory to motivic cohomology, Ann. Sci. École Norm. Sup. (4) 35 (2002), no. 6, 773-875, http://www.math.uiuc.edu/K-theory/0432/.

13. William Fulton, Intersection theory, Ergebnisse der Mathematik und ihrer Grenzgebiete (3) [Results in Mathematics and Related Areas (3)], vol. 2, SpringerVerlag, Berlin, 1984.

14. William Fulton and Serge Lang, Riemann-Roch algebra, Springer-Verlag, New York, 1985.

15. Steven Gersten, K-theory of a polynomial extension, preprint from Rice University, before 1972 .

16. H. Gillet and C. Soulé, Filtrations on higher algebraic K-theory, Algebraic K-theory (Seattle, WA, 1997), Proc. Sympos. Pure Math., vol. 67, Amer. Math. Soc., Providence, RI, 1999, http://www.math.uiuc.edu/K-theory/0327/, pp. 89-148.

17. Daniel R. Grayson, Higher algebraic K-theory. II (after Daniel Quillen), Algebraic K-theory (Proc. Conf., Northwestern Univ., Evanston, Ill., 1976), Springer, Berlin, 1976, pp. 217-240. Lecture Notes in Math., Vol. 551.

18. _ Weight filtrations in algebraic K-theory, Motives (Seattle, WA, 1991), Amer. Math. Soc., Providence, RI, 1994, http://www.math.uiuc.edu/Ktheory/0002/, pp. 207-237.

19. W Weight filtrations via commuting automorphisms, K-Theory 9 (1995), no. 2, 139-172, http://www.math.uiuc.edu/K-theory/0003/.

20. J. P. C. Greenlees and J. P. May, Generalized Tate cohomology, Mem. Amer. Math. Soc. 113 (1995), no. 543, viii+178.

21. A. Grothendieck, Théorie des intersections et théorème de Riemann-Roch, Springer-Verlag, Berlin, 1971, Séminaire de Géométrie Algégrique du Bois-Marie 1966-1967 (SGA 6), Dirigé par P. Berthelot, A. Grothendieck et L. Illusie. Avec la collaboration de D. Ferrand, J. P. Jouanolou, O. Jussila, S. Kleiman, M. Raynaud et J. P. Serre, Lecture Notes in Mathematics, Vol. 225.

22. Bruno Kahn, K-theory of semi-local rings with finite coefficients and étale cohomology, K-Theory 25 (2002), no. 2, 99-138, http://www.math.uiuc.edu/Ktheory $/ 0537 /$.

23. Max Karoubi and Orlando Villamayor, Foncteurs $K^{n}$ en algèbre et en topologie, C. R. Acad. Sci. Paris Sér. A-B 269 (1969), A416-A419.

24. Max Karoubi and Charles Weibel, Algebraic and real K-theory of real varieties, Topology 42 (2003), no. 4, 715-742, http://www.math.uiuc.edu/Ktheory/0473/.

25. Steven E. Landsburg, Some filtrations on higher $K$-theory and related invariants, K-Theory 6 (1992), no. 5, 431-455. 
26. Marc Levine, Bloch's higher Chow groups revisited, Astérisque (1994), no. 226, 10, 235-320, K-theory (Strasbourg, 1992).

27. _ Mixed motives, American Mathematical Society, Providence, RI, 1998.

28. - Techniques of localization in the theory of algebraic cycles, J. Algebraic Geom. 10 (2001), no. 2, 299-363, http://www.math.uiuc.edu/K-theory/0335/.

29. The homotopy coniveau filtration, http://www.math.uiuc.edu/Ktheory/0628/, April 22, 2003.

30. S. Lichtenbaum, Values of zeta-functions at nonnegative integers, Number theory, Noordwijkerhout 1983 (Noordwijkerhout, 1983), Springer, Berlin, 1984, pp. $127-138$.

31. Saunders MacLane, Homology, first ed., Springer-Verlag, Berlin, 1967, Die Grundlehren der mathematischen Wissenschaften, Band 114.

32. Fabien Morel and Vladimir Voevodsky, $\mathbf{A}^{1}$-homotopy theory of schemes, Inst. Hautes Études Sci. Publ. Math. (1999), no. 90, 45-143 (2001), http://www.math.uiuc.edu/K-theory/0305/.

33. Michael Paluch, Topology on $S^{-1} S$ for Banach algebras, Topology 35 (1996), no. 4, 887-900.

34. Claudio Pedrini and Charles Weibel, The higher K-theory of complex varieties, K-Theory 21 (2000), no. 4, 367-385, http://www.math.uiuc.edu/Ktheory/0403/.

35. _ The higher K-theory of a complex surface, Compositio Math. 129 (2001), no. 3, 239-271, http://www.math.uiuc.edu/K-theory/0328/.

36. ㄴ The higher K-theory of real curves, K-Theory 27 (2002), no. 1, 1-31, http://www.math.uiuc.edu/K-theory/0429/.

37. Daniel Quillen, Higher algebraic K-theory. I, Algebraic $K$-theory, I: Higher $K$ theories (Proc. Conf., Battelle Memorial Inst., Seattle, Wash., 1972), Springer, Berlin, 1973, pp. 85-147. Lecture Notes in Math., Vol. 341.

38. Higher algebraic K-theory, Proceedings of the International Congress of Mathematicians (Vancouver, B. C., 1974), Vol. 1, Canad. Math. Congress, Montreal, Que., 1975, pp. 171-176.

39. J. Rognes and C. Weibel, Étale descent for two-primary algebraic $K$-theory of totally imaginary number fields, K-Theory 16 (1999), no. 2, 101-104, http://www.math.uiuc.edu/K-theory/0266/.

40. - Two-primary algebraic K-theory of rings of integers in number fields, J. Amer. Math. Soc. 13 (2000), no. 1, 1-54, Appendix A by Manfred Kolster. http://www.math.uiuc.edu/K-theory/0220/.

41. Andrei Suslin, On the Grayson spectral sequence, http://www.math.uiuc.edu/Ktheory/0588/, August 19, 2002.

42. Vladimir Voevodsky, The Milnor conjecture, http://www.math.uiuc.edu/Ktheory/0170/, December 20, 1996.

43. _ Open problems in the motivic stable homotopy theory, I, http://www.math.uiuc.edu/K-theory/0392/, March 11, 2000.

44. On 2-torsion in motivic cohomology, http://www.math.uiuc.edu/Ktheory/0502/, July 15, 2001.

45. _ Cancellation theorem, http://www.math.uiuc.edu/K-theory/0541/, January 28, 2002.

46. Motivic cohomology groups are isomorphic to higher Chow groups in any characteristic, Int. Math. Res. Not. (2002), no. 7, 351-355, http://www.math.uiuc.edu/K-theory/0378/. 
47. A possible new approach to the motivic spectral sequence for algebraic $K$-theory, Recent progress in homotopy theory (Baltimore, MD, 2000), Contemp. Math., vol. 293, Amer. Math. Soc., Providence, RI, 2002, Preprint available at http://www.math.uiuc.edu/K-theory/0469/, pp. 371-379.

48. On motivic cohomology with $\mathbb{Z} / \ell$-coefficients, http://www.math.uiuc.edu/K-theory/0639/, July 16, 2003.

49. On the zero slice of the sphere spectrum, http://www.math.uiuc.edu/Ktheory/0612/, January 13, 2003.

50. Mark Edward Walker, Motivic complexes and the K-theory of automorphisms, Thesis (Ph. D.), University of Illinois at Urbana-Champaign, available on microfilm from University Microfilms International, 1996.

51. Charles Weibel, The 2-torsion in the K-theory of the integers, C. R. Acad. Sci. Paris Sér. I Math. 324 (1997), no. 6, 615-620, http://www.math.uiuc.edu/Ktheory/0141/. 


\section{Index}

$\gamma$-filtration 3

$\mathbb{S}^{1} \quad 11$

algebraic cycle 3

Atiyah-Hirzebruch spectral sequence

cancellation theorem $\quad 14,15$

Chern character 3

Chern classes 3

Chow group

higher 20

Chow ring 3

commuting automorphisms 9

correspondence 15

direct sum Grothendieck group 15

filtration

of a space 8

Grothendieck group direct sum 11

Grothendieck-Riemann-Roch theorem 3

5 homotopy cofiber 23

homotopy coniveau filtration 25

homotopy fiber 21

layer

of a filtration 8

motivic spectral sequence 1

moving lemma 22,26

multi-relative $K$-theory $\quad 21$

Riemann-Roch theorem 2

slice filtration $\quad 25$

spectrum

motivic 24 
